\title{
Preconditioned Steepest Descent Methods for some Nonlinear Elliptic Equations Involving p-Laplacian Terms
}

\author{
Wenqiang Feng* Abner J. Salgado ${ }^{\dagger} \quad$ Cheng Wang $^{\ddagger} \quad$ Steven M. Wise ${ }^{\S}$
}

December 2, 2016

\begin{abstract}
We describe and analyze preconditioned steepest descent (PSD) solvers for fourth and sixth-order nonlinear elliptic equations that include p-Laplacian terms on periodic domains in 2 and 3 dimensions. The highest and lowest order terms of the equations are constant-coefficient, positive linear operators, which suggests a natural preconditioning strategy. Such nonlinear elliptic equations often arise from time discretization of parabolic equations that model various biological and physical phenomena, in particular, liquid crystals, thin film epitaxial growth and phase transformations. The analyses of the schemes involve the characterization of the strictly convex energies associated with the equations. We first give a general framework for PSD in Hilbert spaces. Based on certain reasonable assumptions of the linear pre-conditioner, a geometric convergence rate is shown for the nonlinear PSD iteration. We then apply the general theory to the fourth and sixth-order problems of interest, making use of Sobolev embedding and regularity results to confirm the appropriateness of our pre-conditioners for the regularized p-Lapacian problems. Our results include a sharper theoretical convergence result for p-Laplacian systems compared to what may be found in existing works. We demonstrate rigorously how to apply the theory in the finite dimensional setting using finite difference discretization methods. Numerical simulations for some important physical application problems - including thin film epitaxy with slope selection and the square phase field crystal model - are carried out to verify the efficiency of the scheme.
\end{abstract}

Keywords: Fourth-order nonlinear elliptic equation, sixth-order nonlinear elliptic equation, p-Laplacian operator, steepest descent, pre-conditioners, finite differences, Fast Fourier transform, thin film epitaxy, square phase field crystal model.

\section{Introduction}

Let $\Omega \subset \mathbb{R}^{d}, d=2,3$, be a rectangular domain. In this work we are interested in efficient solution techniques for fourth and sixth-order nonlinear elliptic equations that have p-Laplacian terms. The fourth-order problem reads as follows: given $f \Omega$-periodic, find $u \Omega$-periodic such that

$$
u-s \nabla \cdot\left(|\nabla u|^{p-2} \nabla u\right)+s \varepsilon^{2} \Delta^{2} u=f
$$

where $0<\varepsilon \leq 1$ and $s$ is a positive parameter. The sixth-order problem is as follows: given $f, g \Omega$-periodic, find $u, w \Omega$-periodic such that

$$
\begin{aligned}
u-\Delta w & =g, \\
s \lambda u-s \nabla \cdot\left(|\nabla u|^{p-2} \nabla u\right)+s \varepsilon^{2} \Delta^{2} u-w & =f,
\end{aligned}
$$

*Department of Mathematics, The University of Tennessee, Knoxville, TN 37996 (wfeng1@vols.utk.edu)

$\dagger$ Department of Mathematics, The University of Tennessee, Knoxville, TN 37996 (asalgad1@utk.edu)

$\ddagger$ Department of Mathematics, The University of Massachusetts, North Dartmouth, MA 02747 (cwang1@umassd.edu)

$\S$ Corresponding author: Department of Mathematics, The University of Tennessee, Knoxville, TN 37996 (swise1@utk.edu)

(C) 2016. This manuscript version is made available under the Elsevier user license http://www.elsevier.com/open-access/userlicense/1.0/ 
where $0<\varepsilon \leq 1, s>0$, and $\lambda \geq 0$ are parameters. The highest order positive diffusion term, parameterized by $\varepsilon$, is often referred to as the surface diffusion, following the thin film applications described below.

We will refer to problems $(1.1)$ and $(1.2 \mathrm{a})-(1.2 \mathrm{~b})$ as regularized $p$-Laplacian problems. However, this is primarily for ease of reference. The highest order surface diffusion term, though parameterized by the "small" coefficient $\varepsilon>0$, must be present for the related physical models to make sense and is not an artificial regularization. In other words, we will not consider and are not concerned with the singular limit $\varepsilon \searrow 0$.

These model equations arise most commonly from the time discretization for certain time-dependent physical models. For example, consider the thin epitaxial film model with slope selection

$$
\partial_{t} u=\nabla \cdot\left(|\nabla u|^{2} \nabla u\right)-\Delta u-\varepsilon^{2} \Delta^{2} u,
$$

in $[28,32,35,38]$, where $u$ is the spatially periodic height of the film. The 4-Laplacian term in combination with the negative Laplacian term gives energetic preference to facets with unit slope, a continuum-level model of the Ehrlich-Schwoebel kinetic barrier. The highest order term models a small amount of surface diffusion, which smooths the corners where the facets merge. In the square Swift-Hohenberg (SSH) equation

$$
\partial_{t} u=(\beta-1) u+\eta u^{3}-u^{5}+\alpha\left(|\nabla u|^{2} \nabla u\right)-2 \Delta u+\Delta^{2} u, \quad \alpha>0, \quad \beta, \eta \in \mathbb{R},
$$

studied in $[11,22,20,30]$, and the square phase field crystal (SPFC) equation

$$
\partial_{t} u=\Delta\left(\gamma_{0} u+\gamma_{1} \Delta u+\varepsilon^{2} \Delta^{2} u-\nabla \cdot\left(|\nabla u|^{2} \nabla u\right)\right), \quad \gamma_{0} \in \mathbb{R}, \quad \gamma_{1}>0,
$$

studied in $[15,19,20,30]$, the 4-Laplacian term gives preference to square-symmetry arrays of "dots" in the density field $u$. In general, such localized structures play important roles in biological, chemical, and physical processes [23].

For these time-dependent problems, convex decomposition schemes have been proposed and analyzed in $[32,35]$ to obtain unconditional unique solvability and unconditional energy stability. The convex decomposition scheme for the thin film model is [35]

$$
u^{m}-s \nabla \cdot\left(\left|\nabla u^{m}\right|^{2} \nabla u^{m}\right)+s \varepsilon^{2} \Delta^{2} u^{m}=u^{m-1}-s \Delta u^{m-1},
$$

where $s>0$ is the time step size, and the superscripts indicate the time discretizations. The convex decomposition scheme for the SPFC model - which can be inferred from the general principles in $[35,37]-$ is precisely

$$
\begin{aligned}
u^{m}-\Delta w^{m} & =u^{m-1}, \\
s \gamma_{0} u^{m}-s \nabla \cdot\left(\left|\nabla u^{m}\right|^{2} \nabla u^{m}\right)+s \varepsilon^{2} \Delta^{2} u^{m}-w^{m} & =-s \gamma_{1} \Delta u^{m-1},
\end{aligned}
$$

assuming $\gamma_{0}, \gamma_{1} \geq 0$. These schemes are nonlinear and require one to deal with the p-Laplacian term at the implicit time level. We remark that there are also second-order-in-time convex decomposition schemes for such nonlinear parabolic equations, as described in [32], which have similar nonlinear structures. In any case, solving nonlinear elliptic equations with the p-Laplacian term is challenging, because of its highly nonlinear nature. In $[32,35]$, the authors used a nonlinear conjugate gradient algorithm to solve the nonlinear system at each implicit time step. Such naive gradient methods are guaranteed to converge due to the global convexity of the equations, but are not necessarily efficient.

Several works develop and analyze numerical schemes for nonlinear elliptic equations involving the $p$-Laplacian operator. The works $[2,3,25,29,34,39,40]$ are based on finite element approximations in space. Recently, the vanishing moment method for the p-Laplacian was proposed in [18]. In that method, the highest order term is purely artificial, whereas, for the models above, the surface diffusion term is small, but non-vanishing. A hybridizable discontinuous Galerkin method for the p-Laplacian was proposed in [10]. Of these works, [25, 39, 40] are primarily focused on efficient solvers for the elliptic equations with $\mathrm{p}$-Laplacian terms, rather than, say, error estimates.

The main goal of this paper is to design a general framework of preconditioned steepest descent (PSD) methods for certain nonlinear elliptic equations with p-Laplacian terms. The main idea is to use a linearized 
version of the nonlinear operator as a pre-conditioner, or in other words, as a metric for choosing the search direction. We propose and analyze the preconditioned steepest descent methods for both the fourth- and sixthorder p-Laplacian problems mentioned above. Herein we present numerical simulations for the 6-Laplacian thin film epitaxy and the $\mathrm{H}^{-1}$ gradient flow SPFC model by using the proposed method. While we restrict our focus to the p-Laplacian problems herein, the search direction framework is general and can be applied to other nonlinear equations, such as the Cahn-Hilliard $(\mathrm{CH})$ equation $[5,7,31,33]$, functionalized Cahn-Hilliard (FCH) Equation $[8,13,16]$, for example.

The convergence analyses of the nonlinear iteration algorithms we propose for the p-Laplacian equations are quite challenging, due to the highly nonlinear nature of the problems. However, we are able to recast the equations as equivalent minimization problems involving strictly convex functionals in Hilbert spaces. Once this is done, we are able to characterize the properties of general pre-conditioners that will result in geometric convergence rates. This general approach is applicable to both the 4th and 6th order equations at the space-continuous level, as well as the approximation of these problems in finite dimensions using finite differences. Though we do not explore it here, we remark that the theory is extensible to the pseudo-spectral, spectral-Galerkin, and mixed finite element settings as well, using the appropriate discrete Gagliardo-Nirenburg inequalities. To our knowledge, the only related theoretical results available in the existing literature are to be found in [25], in which finite element PSD solvers were designed and analyzed. Specifically, it was proved in [25] that their method converges with the rate $O\left(k^{-\beta}\right)$, where $k$ is the iteration index and $\beta=\frac{p}{p-2}>0$. In this article, we provide a theoretical analysis with a geometric convergence rate $O\left(\alpha^{k}\right)$, with $0<\alpha<1$, for the finite difference PSD solver applied to the regularized p-Laplacian problems.

For such nonlinear analyses, the essential difficulty has always been associated with the subtle fact that the numerical solution has to be bounded uniformly in certain functional norms, so that a bound for the iteration error could be established. For the $\mathrm{p}$-Laplacian problems, typically a uniform $W^{1, p}$ bound of the numerical solution is available at each iteration stage, and such a bound may be used to derive an $O\left(k^{-\beta}\right)$ convergence rate for the PSD iteration. However, for the regularized p-Laplacian problems, one observes that a linear operator with higher-order diffusion may be utilized so that a uniform $\mathrm{H}^{2}$ bound of the numerical solution may be obtained. Specifically, the existence of the surface diffusion term $\varepsilon^{2} \Delta^{2} u$ enables us to derive a geometric convergence rate $O\left(\alpha^{k}\right)$ for the PSD iteration, which gives a sharper theoretical result than the existing one in [25]. Our strategy comes at a cost that we point out at the offset: a linear, positive, constant-coefficient operator of order 4 or 6 must be inverted to obtain the search direction. But, since we are interested in applications involving coarsening processes over periodic domains, the FFT can be utilized to make this process efficient.

The remainder of the paper is organized as follows. In section 2, we present a general preconditioned steepest descent (PSD) method for nonlinear equations in Hilbert spaces, and provide the convergence rate estimates for the PSD method. The application of the general theory to the fourth-order regularized p-Laplacian problem is formulated in section 3. The PSD scheme for the sixth-order regularized p-Laplacian problem is outlined in section 4. Subsequently, in section 5, we introduce a two-dimensional finite difference discretization and provide the fully discrete convergence analysis. Applications to thin film epitaxy with slope selection and the SPFC model and the numerical results are presented in section 6. Concluding remarks are offered in section 7.

\section{Preconditioned Steepest Descent Methods}

\subsection{Non-Quadratic Energy Functionals in Hilbert Spaces}

Here we outline the general theory for preconditioned steepest descent (PSD) in a Hilbert space $[1,9,14,26]$. Suppose that $H$ is a (real) Hilbert space with the inner product $(\cdot, \cdot)_{H}$ and induced norm $\|\cdot\|_{H}$. We consider an energy functional $E[\cdot]: H \rightarrow \mathbb{R}$ with the following properties:

(E1) $E$ is twice Fréchet differentiable for all points $\nu \in H$. For each fixed $\nu \in H, \delta E[\nu](\cdot): H \rightarrow \mathbb{R}$ is the continuous linear functional equal to the first Fréchet derivative at $\nu$, and, for each fixed $\nu \in H$, $\delta^{2} E[\nu](\cdot, \cdot): H \times H \rightarrow \mathbb{R}$ is the continuous bilinear operator equal to the second Fréchet derivative at $\nu$. 
(E2) For every $\nu \in H$,

$$
0 \leq \delta^{2} E[\nu](\xi, \xi), \quad \forall \xi \in H
$$

and

$$
0<\delta^{2} E[\nu](\xi, \xi), \quad \forall \xi \in H \backslash\{0\} .
$$

This implies the strict convexity of $E$.

(E3) $E$ is coercive with respect to the norm on $H$, i.e., there exist constants $C_{1}>0, C_{2} \geq 0$ such that

$$
C_{1}\|\nu\|_{H}^{2} \leq E[\nu]+C_{2}, \quad \forall \nu \in H .
$$

If $E$ satisfies (E1) - (E3), it follows [9] that there is a unique element $u \in H$ with the property that

$$
E[u] \leq E[\nu], \quad \forall \nu \in H, \quad \text { with } \quad E[u]<E[\nu], \quad \text { for } \nu \neq u,
$$

and this minimizer further satisfies

$$
\delta E[u](\xi)=0, \quad \forall \xi \in H .
$$

We wish to construct, via preconditioned steepest descent (PSD), a sequence that converges to the unique minimizer. By $H^{\prime}$ we denote the continuous dual of $H$. When it is convenient, we use the symbol $\langle\cdot, \cdot\rangle_{H}$ : $H^{\prime} \times H \rightarrow \mathbb{R}$ to denote the dual pairing between $H^{\prime}$ and $H$. Consider a linear operator $\mathcal{L}: H \rightarrow H^{\prime}$. This operator $\mathcal{L}$, which we call the pre-conditioner induces a bilinear form on $H$ :

$$
(\nu, \xi)_{\mathcal{L}}:=\langle\mathcal{L}[\nu], \xi\rangle_{H}=\mathcal{L}[\nu](\xi), \quad \forall \nu, \xi \in H .
$$

We assume that $\mathcal{L}$ satisfies the following properties:

(L1) $(\cdot, \cdot)_{\mathcal{L}}: H \times H \rightarrow \mathbb{R}$ is symmetric, i.e.,

$$
(\nu, \xi)_{\mathcal{L}}=(\xi, \nu)_{\mathcal{L}}, \quad \forall \nu, \xi \in H
$$

(L2) $(\cdot, \cdot)_{\mathcal{L}}$ is continuous with respect to the standard topology of $H$, i.e., there is some $C_{3}>0$ such that

$$
\left|(\nu, \xi)_{\mathcal{L}}\right| \leq C_{3}\|\nu\|_{H}\|\xi\|_{H}, \quad \forall \nu, \xi \in H ;
$$

(L3) $(\cdot, \cdot)_{\mathcal{L}}$ is coercive with respect to $H$, i.e., there is some $C_{4}>0$ such that

$$
C_{4}\|\nu\|_{H}^{2} \leq(\nu, \nu)_{\mathcal{L}}, \quad \forall \nu \in H .
$$

It follows that $(\cdot, \cdot)_{\mathcal{L}}: H \times H \rightarrow \mathbb{R}$ is an inner product on $H$, equivalent to the primary inner product $(\cdot, \cdot)_{H}$. The induced norm, $\|\nu\|_{\mathcal{L}}:=\sqrt{(\nu, \nu)_{\mathcal{L}}}$, is equivalent to the primary norm. By the Riesz Representation Theorem, if $f \in H^{\prime}$, then there exists a unique $u_{f} \in H$ such that

$$
\left(u_{f}, \xi\right)_{\mathcal{L}}=f[\xi]=\langle f, \xi\rangle_{H}, \quad \forall \xi \in H,
$$

with

$$
\left\|u_{f}\right\|_{\mathcal{L}}=\|f\|_{\mathcal{L}^{-1}}:=\sup _{\substack{0 \neq \xi \in H\\}} \frac{f[\xi]}{\|\xi\|_{\mathcal{L}}}
$$

where the second norm is the $\mathcal{L}$-induced operator norm.

Suppose that $u^{k} \in H$ is given. We define the following search direction problem: find $d^{k} \in H$ such that

$$
\left(d^{k}, \xi\right)_{\mathcal{L}}=-\delta E\left[u^{k}\right](\xi), \quad \forall \xi \in H .
$$

We call $d^{k}$ the $k^{\text {th }}$ search direction. In operator form, we write $\mathcal{L}\left[d^{k}\right]=-\delta E\left[u^{k}\right]$ in $H^{\prime}$. The functional $-\delta E\left[u^{k}\right]$ is called the residual of $u^{k}$. By the Riesz Representation Theorem, we discover that

$$
-\delta E\left[u^{k}\right]\left(d^{k}\right)=\left\|d^{k}\right\|_{\mathcal{L}}^{2}=\left\|\delta E\left[u^{k}\right]\right\|_{\mathcal{L}^{-1}}^{2} .
$$


We then define the next iterate $u^{k+1}$ as

$$
u^{k+1}:=u^{k}+\alpha_{k} d^{k}
$$

where $\alpha_{k} \in \mathbb{R}$ is the unique solution to

$$
\alpha_{k}:=\underset{\alpha \in \mathbb{R}}{\operatorname{argmin}} E\left[u^{k}+\alpha d^{k}\right]=\underset{\alpha \in \mathbb{R}}{\operatorname{argzero}} \delta E\left[u^{k}+\alpha d^{k}\right]\left(d^{k}\right) .
$$

Therefore, we have the fundamental orthogonality relation

$$
\delta E\left[u^{k}+\alpha_{k} d^{k}\right]\left(d^{k}\right)=\delta E\left[u^{k+1}\right]\left(d^{k}\right)=0 .
$$

It follows that the sequence $\left\{u^{k}\right\}_{k=0}^{\infty} \subset H$ generated by the preconditioned steepest descent algorithm converges to the unique minimizer $u \in H$. We now wish to estimate the convergence rate.

\subsection{Estimates of the Convergence Rate for the PSD Method}

We summarize some standard results.

Proposition 2.1. Suppose that $E$ satisfies $(E 1)-(E 3)$. It follows that, for any $\nu, \xi \in H$,

$$
\delta E[\nu](\xi-\nu) \leq E[\xi]-E[\nu] \leq \delta E[\xi](\xi-\nu)
$$

and, consequently,

$$
0 \leq(\delta E[\xi]-\delta E[\nu])(\xi-\nu)
$$

Proposition 2.2. Suppose that $E$ satisfies (E1) - (E3). Let $\left\{u^{k}\right\}_{k=0}^{\infty} \subset H$ be computed via (2.5). Then, for every $k \geq 0$ we have $E\left[u^{k+1}\right] \leq E\left[u^{k}\right]$. Furthermore, $\alpha_{k}>0$, as long as $u^{k} \neq u$.

Proof. Using the orthogonality relation (2.7) and the convexity inequality (2.8), we find

$$
E\left[u^{k+1}\right]-E\left[u^{k}\right] \leq \delta E\left[u^{k+1}\right]\left(u^{k+1}-u^{k}\right)=\alpha_{k} \delta E\left[u^{k+1}\right]\left(d^{k}\right)=0 .
$$

Now, suppose $d^{k} \neq 0$. Then, by Taylor's theorem, (2.4), and (2.2),

$$
E\left[u^{k+1}\right]=E\left[u^{k}\right]-\alpha_{k}\left\|d^{k}\right\|_{\mathcal{L}}^{2}+\frac{\alpha_{k}^{2}}{2} \delta^{2} E\left[\theta^{k}\right]\left(d^{k}, d^{k}\right)>E\left[u^{k}\right]-\alpha_{k}\left\|d^{k}\right\|_{\mathcal{L}}^{2} .
$$

Equivalently, we get

$$
\alpha_{k}\left\|d^{k}\right\|_{\mathcal{L}}^{2}>E\left[u^{k}\right]-E\left[u^{k+1}\right] \geq 0
$$

which implies that $\alpha_{k}>0$.

Proposition 2.3. Suppose that $E$ satisfies $(E 1)-(E 3)$ and $u \in H$ is the unique minimizer of $E$. Then, for any $\xi \in H$,

$$
0 \leq E[\xi]-E[u] \leq(\delta E[\xi]-\delta E[u])(\xi-u)=\delta E[\xi](\xi-u)
$$

and, consequently,

$$
0 \leq E\left[u^{k}\right]-E[u] \leq\left(\delta E\left[u^{k}\right]-\delta E[u]\right)\left(u^{k}-u\right)=\delta E\left[u^{k}\right]\left(u^{k}-u\right)
$$

Proof. This follows immediately from (2.8), because $\delta E[u](\xi)=0$, for all $\xi \in H$.

Now, we make the following further assumptions about the pre-conditioner $\mathcal{L}$ with respect to the derivatives of the energy $E$ :

(L4) There is a constant $C_{5}>0$ such that

$$
C_{5}\|\xi-\nu\|_{\mathcal{L}}^{2} \leq(\delta E[\xi]-\delta E[\nu])(\xi-\nu)
$$

for all $\nu, \xi \in H$. 
(L5) Suppose $B:=\left\{\nu \in H \mid E[\nu] \leq E_{0}\right\}$ is non-empty. (This is the the case if, for example, one chooses $E_{0}=E[0]$.) There is a constant $C_{6}=C_{6}\left(E_{0}\right)>0$ such that, for all $\nu \in B$, and any $\xi \in H$,

$$
\left|\delta^{2} E[\nu](\xi, \xi)\right| \leq C_{6}\|\xi\|_{\mathcal{L}}^{2} .
$$

Remark 2.4. We note that, practically speaking, (L5) is harder of the last two conditions to enforce. In some sense, if the norm induced by $\mathcal{L}$ is not "strong" enough, then there does not exist $C_{6}>0$ so that (L5) is satisfied.

Theorem 2.5. Suppose that assumptions (E1) - (E3) and (L1) - (L5) are valid. Let $\left\{u^{k}\right\}_{k=0}^{\infty} \subset H$ be the sequence generated by (2.5). Then

$$
0 \leq E\left[u^{k}\right]-E[u] \leq\left(C_{7}\right)^{k}\left(E\left[u^{0}\right]-E[u]\right),
$$

where

$$
0<C_{7}:=1-\frac{C_{5}}{2 C_{6}}<1
$$

Proof. Consider the function $g(\alpha):=E\left[u^{k}+\alpha d^{k}\right]-E\left[u^{k}\right], \alpha \in \mathbb{R}$. Then $g(0)=0$, and $g$ has a global minimum at $\alpha_{k}>0$. By coercivity and continuity of $E$, there is a $\beta_{k}, \alpha_{k}<\beta_{k}<\infty$, such that $g\left(\beta_{k}\right)=0$, and, for all $\alpha \in\left[0, \beta_{k}\right]$,

$$
E\left[u^{k}+\alpha d^{k}\right] \leq E\left[u^{k}\right] \leq E\left[u^{0}\right]=: E_{0} .
$$

By Taylor's theorem, there is a $\gamma=\gamma\left(u^{k}, d^{k}, \alpha\right) \in(0,1)$, such that

$$
E\left[u^{k}+\alpha d^{k}\right]-E\left[u^{k}\right]=\alpha \delta E\left[u^{k}\right]\left(d^{k}\right)+\frac{\alpha^{2}}{2} \delta^{2} E\left[\theta^{k}\right]\left(d^{k}, d^{k}\right),
$$

where $\theta^{k}:=u^{k}+(1-\gamma) \alpha d^{k}$. By convexity of $E$,

$$
E\left[\theta^{k}\right] \leq \gamma E\left[u^{k}\right]+(1-\gamma) E\left[u^{k}+\alpha d^{k}\right] \leq E\left[u^{k}\right] \leq E\left[u^{0}\right]=E_{0} .
$$

Using estimate (2.11) - with the set $B$ defined with respect to $E_{0}=E\left[u^{0}\right]$ - and norm equality (2.4), we get, for all $\alpha \in\left[0, \beta_{k}\right]$,

$$
\begin{aligned}
g(\alpha)=E\left[u^{k}+\alpha d^{k}\right]-E\left[u^{k}\right] & \leq \alpha \delta E\left[u^{k}\right]\left(d^{k}\right)+\frac{\alpha^{2}}{2} C_{6}\left\|d^{k}\right\|_{\mathcal{L}}^{2} \\
& =\left(-\alpha+\frac{\alpha^{2}}{2} C_{6}\right)\left\|\delta E\left[u^{k}\right]\right\|_{\mathcal{L}^{-1}}^{2}=: f(\alpha) .
\end{aligned}
$$

Now, the function $f(\alpha)$ is quadratic, $f(0)=0, f\left(\beta_{k}\right) \geq g\left(\beta_{k}\right)=0$, and $f^{\prime}(0)<0$. See Figure 1 . Thus $f$ has a minimum in $\left(0, \beta_{k}\right)$. In fact, the minimum is achieved at $0<\sigma_{k}:=\frac{1}{C_{6}}<\beta_{k}$. Then we have

$$
E\left[u^{k}+\alpha_{k} d^{k}\right]-E\left[u^{k}\right] \leq g\left(\sigma_{k}\right)=E\left[u^{k}+\sigma_{k} d^{k}\right]-E\left[u^{k}\right] \leq-\frac{1}{2 C_{6}}\left\|\delta E\left[u^{k}\right]\right\|_{\mathcal{L}^{-1}}^{2}=f\left(\sigma_{k}\right),
$$

or, equivalently,

$$
E\left[u^{k}\right]-E\left[u^{k+1}\right] \geq \frac{1}{2 C_{6}}\left\|\delta E\left[u^{k}\right]\right\|_{\mathcal{L}^{-1}}^{2} .
$$

Now, using estimates (2.9) and (2.10) we obtain

$$
0 \leq E\left[u^{k}\right]-E[u] \leq \frac{1}{C_{5}}\left\|\delta E\left[u^{k}\right]\right\|_{\mathcal{L}^{-1}}^{2} .
$$

Combining the last two estimates, we get the result

$$
0 \leq E\left[u^{k}\right]-E[u] \leq \frac{2 C_{6}}{C_{5}}\left(E\left[u^{k}\right]-E\left[u^{k+1}\right]\right),
$$




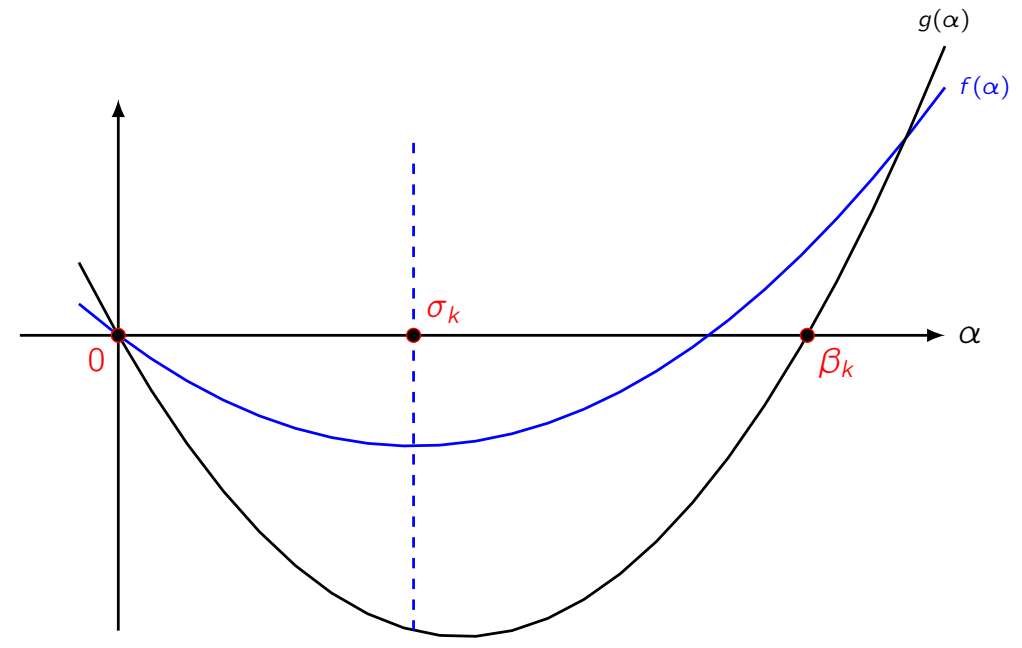

Figure 1: The functions $g(\alpha)=E\left[u^{k}+\alpha d^{k}\right]-E\left[u^{k}\right]$ and $f(\alpha)=\left(-\alpha+\frac{\alpha^{2}}{2} C_{6}\right)\left\|\delta E\left[u^{k}\right]\right\|_{\mathcal{L}^{-1}}^{2}$ from (2.14). The function $g$, which is strictly convex, is dominated by the function $f$, which is quadratic, on the interval $\left[0, \beta_{k}\right]$.

or, equivalently,

$$
0 \leq E\left[u^{k+1}\right]-E[u] \leq\left(\frac{2 C_{6}}{C_{5}}-1\right)\left(E\left[u^{k}\right]-E\left[u^{k+1}\right]\right)
$$

Since $E\left[u^{k+1}\right]>E[u]$, as long as $u^{k+1} \neq u$, and $E\left[u^{k}\right] \geq E\left[u^{k+1}\right]$, this last inequality implies that

$$
0<\frac{C_{5}}{2 C_{6}}<1
$$

A little more manipulation reveals the equivalent inequality

$$
0 \leq E\left[u^{k+1}\right]-E[u] \leq\left(1-\frac{C_{5}}{2 C_{6}}\right)\left(E\left[u^{k}\right]-E[u]\right),
$$

and the result follows.

If the following property holds, we get a simple corollary of the last theorem.

(L6) There is a constant $C_{8}>0$, such that, for every $\nu, \xi \in H$,

$$
C_{8}\|\xi\|_{\mathcal{L}}^{2} \leq\left|\delta^{2} E[\nu](\xi, \xi)\right| .
$$

This implies the strong convexity of $E$ and is, therefore, stronger that (E2).

Corollary 2.6. Suppose that assumptions (E1) - (E3) and $(L 1)-(L 6)$ are valid. Let $\left\{u^{k}\right\}_{k=0}^{\infty} \subset H$ be the sequence generated by (2.5), and define $e^{k}:=u-u^{k}$. Then

$$
\left\|e^{k}\right\|_{\mathcal{L}}^{2} \leq\left(C_{7}\right)^{k} \frac{E\left[u^{0}\right]-E[u]}{C_{8}} .
$$

Proof. By Taylor's theorem and estimate (2.15), we have

$$
\begin{aligned}
E\left[u^{k}\right]-E[u] & =\delta E[u]\left(e^{k}\right)+\frac{1}{2} \delta^{2} E\left[\theta^{k}\right]\left(e^{k}, e^{k}\right) \\
& =\frac{1}{2} \delta^{2} E\left[\theta^{k}\right]\left(e^{k}, e^{k}\right) \geq C_{8}\left\|e^{k}\right\|_{\mathcal{L}}^{2},
\end{aligned}
$$

where $\theta^{k}$ is in the line segment from $u^{k}$ to $u$. The result follows from (2.12). 


\section{Nonlinear Elliptic Equations on Periodic Domains}

\subsection{Notation for Periodic Sobolev Spaces}

For the remainder of paper $\Omega \subset \mathbb{R}^{d}$ with $d=2,3$ is a rectangular domain. In what follows, if $d=2$ we assume $p \in[2, \infty)$; whereas if $d=3$ we suppose $p \in[2,6]$. Most of the physically relevant cases correspond to $p$ being an even integer, however, all of our arguments hold for any value of $p$ in the indicated ranges. The Sobolev spaces of periodic functions are defined as follows: for $q \in[1, \infty]$, we set

$$
W_{\mathrm{per}}^{k, q}(\Omega):=\left\{u \in W_{\mathrm{loc}}^{k, q}\left(\mathbb{R}^{d}\right) \mid u \text { is } \Omega-\text { periodic }\right\},
$$

where $k \in \mathbb{N}$ is the differentiability index. Observe that $W_{\text {per }}^{0, q}(\Omega)=: L_{\text {per }}^{q}(\Omega)=L^{q}(\Omega)$. We denote the norm of $W_{\text {per }}^{k, q}(\Omega)$ by $\|\cdot\|_{W^{k, q},}$, or just $\|\cdot\|_{L^{q}}$ when $k=0$. In the case $q=2$ and $k=0$, we denote by $(\cdot, \cdot)$ and $\|\cdot\|$ the inner product and norm, respectively. We set $H_{\text {per }}^{k}(\Omega)=W_{\text {per }}^{k, 2}(\Omega)$ and immediately remark that, given the range of $p$, we have $H_{\text {per }}^{2}(\Omega) \hookrightarrow W_{\text {per }}^{1, p}(\Omega)$. For $k \in \mathbb{N}$, the continuous dual of $H_{\text {per }}^{k}(\Omega)$ is denoted by $H_{\text {per }}^{-k}(\Omega)$ and their pairing by $\langle\cdot, \cdot\rangle_{k}$. We set $\langle\cdot, \cdot\rangle:=\langle\cdot, \cdot\rangle_{1}$.

If $L_{0}^{2}(\Omega)$ denotes the set of functions in $L^{2}(\Omega)$ with zero mean, we define

$$
\stackrel{\circ}{\text { per }}^{1}(\Omega):=H_{\text {per }}^{1}(\Omega) \cap L_{0}^{2}(\Omega), \quad \stackrel{H}{\text { per }}^{-1}(\Omega):=\left\{v \in H_{\text {per }}^{-1}(\Omega) \mid\langle v, 1\rangle=0\right\}
$$

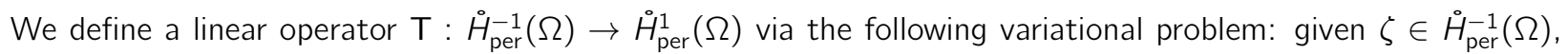
$\mathrm{T}[\zeta] \in \stackrel{H}{\mathrm{per}}^{1}(\Omega)$ solves

$$
(\nabla \mathrm{T}[\zeta], \nabla \chi)=\langle\zeta, \chi\rangle, \quad \forall \chi \in \stackrel{\circ}{\mathrm{per}}^{1}(\Omega) .
$$

From the Riesz representation theorem it immediately follows that $\mathrm{T}$ is well-defined. We define the inner product

$$
(\zeta, \xi)_{\stackrel{H}{\mathrm{per}}^{-1}}:=(\nabla \mathrm{T}[\zeta], \nabla \mathrm{T}[\xi])=\langle\zeta, \mathrm{T}[\xi]\rangle=\langle\xi, \mathrm{T}[\zeta]\rangle, \quad \forall \zeta, \xi \in \stackrel{\circ}{\mathrm{per}}_{\mathrm{per}}^{-1}(\Omega)
$$

The induced norm is denoted $\|\cdot\|_{\dot{H}_{\mathrm{per}}^{-1}}$. The following facts can be easily established [12]:

Lemma 3.1. On $\stackrel{\circ}{\mathrm{per}}_{\mathrm{per}}^{-1}(\Omega)$ the norm $\|\cdot\|_{\stackrel{\circ}{\mathrm{per}}^{-1}}$ equals the operator norm: for all $\zeta \in \stackrel{\circ}{\mathrm{p}}_{\text {per }}^{-1}(\Omega)$,

$$
\|\zeta\|_{\hat{H}_{\mathrm{per}}^{-1}}=\sup _{0 \neq \chi \in \dot{H}_{\mathrm{per}}^{1}(\Omega)} \frac{\langle\zeta, \chi\rangle}{\|\nabla \chi\|} .
$$

Consequently, we have $|\langle\zeta, \chi\rangle| \leq\|\zeta\|_{\stackrel{H}{\text { per }}^{-1}}\|\nabla \chi\|$, for all $\chi \in H_{\text {per }}^{1}(\Omega)$ and $\zeta \in \stackrel{\circ}{\text { per }}^{-1}(\Omega)$. Furthermore, for all $\zeta \in L_{0}^{2}(\Omega)$, we have the Poincaré type inequality: $\|\zeta\|_{\stackrel{H}{\mathrm{per}}^{-1}} \leq C\|\zeta\|$, for some $C>0$.

\subsection{A Fourth-Order Regularized p-Laplacian Problem}

We consider the following weak formulation of (1.1): given $f \in L_{\text {per }}^{2}(\Omega)$, find $u \in H_{\text {per }}^{2}(\Omega)$ such that

$$
(u, \xi)+s\left(|\nabla u|^{p-2} \nabla u, \nabla \xi\right)+s \varepsilon^{2}(\Delta u, \Delta \xi)=(f, \xi), \quad \forall \xi \in H_{\text {per }}^{2}(\Omega),
$$

where $0<\varepsilon \leq 1$ and $s>0$ are parameters. Equation (3.1) is mass conservative in the following sense: $(u-f, 1)=0$. One can show that the solution of the weak formulation is a minimizer of the following energy: for any $\nu \in H_{\text {per }}^{2}(\Omega)$,

$$
E[\nu]:=\frac{1}{2}\|\nu-f\|^{2}+\frac{s}{p}\|\nabla \nu\|_{L^{p}}^{p}+\frac{s \varepsilon^{2}}{2}\|\Delta \nu\|^{2} .
$$

It is not difficult to show that $E$ satisfies $(E 1)-(E 3)$. The first derivative of $E$ at a point $\nu$ may be calculated as follows: for any $\xi \in H_{\text {per }}^{2}(\Omega)$,

$$
\left.d_{\tau} E[\nu+\tau \xi]\right|_{\tau=0}=\delta E[\nu](\xi)=(\nu-f, \xi)+s\left(|\nabla \nu|^{p-2} \nabla \nu, \nabla \xi\right)+s \varepsilon^{2}(\Delta \nu, \Delta \xi)
$$


Thus, our original problem is equivalent to the following: find $u \in H_{\text {per }}^{2}(\Omega)$, such that, for all $\xi \in H_{\text {per }}^{2}(\Omega)$, $\delta E[u](\xi)=0$, which is equivalent to (3.1). This problem has a unique solution, which is, in turn, the unique minimizer of the energy (3.2):

$$
u:=\underset{\nu \in H_{\text {per }}^{2}(\Omega)}{\operatorname{argmin}} E[\nu] .
$$

The following estimate holds: for all $\nu, \xi \in H_{\text {per }}^{2}(\Omega)$,

$$
|\delta E[\nu](\xi)| \leq\|\nu-f\| \cdot\|\xi\|+s\|\nabla \nu\|_{L^{p}}^{p-1}\|\nabla \xi\|_{L^{p}}+s \varepsilon^{2}\|\Delta \nu\| \cdot\|\Delta \xi\| .
$$

The second variation is a continuous bilinear operator. Given a fixed $\nu \in H_{\mathrm{per}}^{2}(\Omega)$, the action of the second variation on the arbitrary pair $(\xi, \eta) \in H_{\text {per }}^{2}(\Omega) \times H_{\text {per }}^{2}(\Omega)$ is given by

$$
\begin{aligned}
\delta^{2} E[\nu](\xi, \eta)= & (\xi, \eta)+s\left(|\nabla \nu|^{p-2} \nabla \xi, \nabla \eta\right) \\
& +(p-2) s\left(|\nabla \nu|^{p-4} \nabla \nu \cdot \nabla \xi, \nabla \nu \cdot \nabla \eta\right)+s \varepsilon^{2}(\Delta \xi, \Delta \eta),
\end{aligned}
$$

and we have the bound

$$
\begin{aligned}
\left|\delta^{2} E[\nu](\xi, \eta)\right| \leq & \|\xi\| \cdot\|\eta\|+s\|\nabla \nu\|_{L^{p}}^{p-2}\|\nabla \xi\|_{L^{p}}\|\nabla \eta\|_{L^{p}} \\
& +(p-2) s\|\nabla \nu\|_{L^{p}}^{p-2}\|\nabla \xi\|_{L^{p}}\|\nabla \eta\|_{L^{p}}+s \varepsilon^{2}\|\Delta \xi\| \cdot\|\Delta \eta\| .
\end{aligned}
$$

For this problem we define the pre-conditioner $\mathcal{L}: H_{\text {per }}^{2}(\Omega) \rightarrow H_{\text {per }}^{-2}(\Omega)$ via

$$
\langle\mathcal{L}[\nu], \xi\rangle:=(\nu, \xi)+s(\nabla \nu, \nabla \xi)+s \varepsilon^{2}(\Delta \nu, \Delta \xi), \quad \forall \xi \in H_{\text {per }}^{2}(\Omega) .
$$

Clearly, this is a positive, symmetric operator, and it satisfies assumptions (L1) - (L3), and one can see the similarities with the nonlinear operator in (3.1). We now proceed to find the positive constants for which $C_{5}, C_{6}, C_{8}$ assumptions (L4) $-(\mathrm{L} 6)$ are satisfied.

Remark 3.2. We could also consider the possibility of changing the metric in the descent direction calculation by, for example, defining the linear operator $\mathcal{L}_{k}: H_{\text {per }}^{2}(\Omega) \rightarrow H_{\text {per }}^{-2}(\Omega)$ via

$$
\left\langle\mathcal{L}_{k}[\nu], \xi\right\rangle:=(\nu, \xi)+s\left(\left|\nabla u^{k}\right|^{p-2} \nabla \nu, \nabla \xi\right)+s \varepsilon^{2}(\Delta \nu, \Delta \xi), \quad \forall \xi \in H_{\mathrm{per}}^{2}(\Omega) .
$$

This is similar to the idea in [26]. The search direction is then found as follows: find $d^{k} \in H_{\text {per }}^{2}(\Omega)$ such that

$$
\left\langle\mathcal{L}_{k}\left[d^{k}\right], \xi\right\rangle=-\delta E\left[u^{k}\right](\xi), \quad \forall \xi \in H_{\text {per }}^{2}(\Omega) .
$$

Our theory does not cover this case, and we will not consider it further here. We plan to examine this in a future work.

Lemma 3.3. Suppose that $p \in[2, \infty)$ when $d=2$, and $p \in[2,6]$, if $d=3$. For any $\xi \in H_{\mathrm{per}}^{2}(\Omega)$, we have

$$
\|\nabla \xi\|_{L^{p}} \leq C_{9}\left\{\begin{array}{lll}
\|\xi\|^{\frac{1}{p}} \cdot\|\Delta \xi\|^{\frac{p-1}{p}}, & \text { if } \quad d=2, & p \in[2, \infty), \\
\|\xi\|^{\frac{3}{2 p}-\frac{1}{4}} \cdot\|\Delta \xi\|^{\frac{5}{4}-\frac{3}{2 p}}, & \text { if } \quad d=3, \quad p \in[2,6],
\end{array}\right.
$$

for some $C_{9}=C_{9}(d, p)>0$.

Proof. This follows from the Gagliardo-Nirenberg interpolation inequality and elliptic regularity.

Lemma 3.4. For any $\nu, \xi \in H_{\mathrm{per}}^{2}(\Omega)$,

$$
C_{5}\|\xi-\nu\|_{\mathcal{L}}^{2} \leq(\delta E[\xi]-\delta E[\nu])(\xi-\nu)
$$

where $C_{5}=\min \left(\frac{1}{2}, \varepsilon s^{-\frac{1}{2}}\right)$. Let $E_{0}$ be given, such that $B:=\left\{\nu \in H_{\mathrm{per}}^{2}(\Omega) \mid E[\nu] \leq E_{0}\right\}$ is non-empty. For any $\nu \in B$ and any $\xi \in H_{\text {per }}^{2}(\Omega)$,

$$
\left|\delta^{2} E[\nu](\xi, \xi)\right| \leq C_{6}\|\xi\|_{\mathcal{L}}^{2}
$$


where

$$
C_{6}=\left\{\begin{array}{lll}
1+\frac{1}{p}(p-1)^{\frac{2 p-1}{p}} \varepsilon^{\frac{-2(p-1)}{p}} S^{\frac{1}{p}} C_{9}^{2} C_{10}^{p-2} & \text { for } p \in[2, \infty), & d=2, \\
1+(p-1)\left(\frac{4 p}{6-p}\right)^{\frac{p-6}{4 p}}\left(\frac{4 p}{5 p-6}\right)^{\frac{6-5 p}{4 p}} s^{\frac{6-p}{4 p}} \varepsilon^{\frac{6-5 p}{2 p}} C_{9}^{2} C_{10}^{p-2} & \text { for } p \in[2,6), & d=3, \\
1+(p-1) \varepsilon^{-2} C_{9}^{2} C_{10}^{p-2} & \text { for } p=6, & d=3,
\end{array}\right.
$$

and $C_{10}=\left(p E_{0}\right)^{\frac{1}{p}}$. We can take $C_{8}=C_{5}$ to satisfy estimate (2.15) of assumption (L6).

Proof. Clearly

$$
\begin{aligned}
(\delta E[\xi]-\delta E[\nu])(\xi-\nu)= & \|\xi-\nu\|^{2}+s \varepsilon^{2}\|\Delta(\xi-\nu)\|^{2} \\
& +s\left(|\nabla \xi|^{p-2} \nabla \xi-|\nabla \nu|^{p-2} \nabla \nu, \nabla(\xi-\nu)\right) .
\end{aligned}
$$

In addition, the following estimate is available:

$$
\left(|\nabla \xi|^{p-2} \nabla \xi-|\nabla \nu|^{p-2} \nabla \nu, \nabla(\xi-\nu)\right) \geq \frac{1}{2^{p-2}}\|\nabla(\xi-\nu)\|_{L^{p}}^{p} \geq 0, \quad \text { for } p \geq 2 .
$$

The simple interpolation inequality

$$
\|\nabla \xi\|^{2} \leq\|\xi\| \cdot\|\Delta \xi\|, \quad \forall \xi \in H_{\mathrm{per}}^{2}(\Omega),
$$

in conjunction with Young's inequality yields

$$
\frac{1}{2}\|\xi-\nu\|^{2}+\frac{s \varepsilon^{2}}{2}\|\Delta(\xi-\nu)\|^{2} \geq s^{\frac{1}{2}} \varepsilon\|\xi-\nu\| \cdot\|\Delta(\xi-\nu)\| \geq s^{\frac{1}{2}} \varepsilon\|\nabla(\xi-\nu)\|^{2} .
$$

As a consequence, we get

$$
\begin{aligned}
(\delta E[\xi]-\delta E[\nu])(\xi-\nu) & \geq\|\xi-\nu\|^{2}+s \varepsilon^{2}\|\Delta(\xi-\nu)\|^{2} \\
& \geq \frac{1}{2}\|\xi-\nu\|^{2}+\frac{1}{2} s \varepsilon^{2}\|\Delta(\xi-\nu)\|^{2}+s^{\frac{1}{2}} \varepsilon\|\nabla(\xi-\nu)\|^{2},
\end{aligned}
$$

and we conclude that estimate (3.5) is valid by choosing $C_{5}=\min \left(\frac{1}{2}, \varepsilon s^{-\frac{1}{2}}\right)$.

Next we derive (3.6). Suppose $\nu \in B$. From (3.3) we have

$$
\left|\delta^{2} E[\nu](\xi, \xi)\right| \leq\|\xi\|^{2}+(p-1) s\|\nabla \nu\|_{L^{p}}^{p-2}\|\nabla \xi\|_{L^{p}}^{2}+s \varepsilon^{2}\|\Delta \xi\|^{2} .
$$

Now, since $\nu \in B$,

$$
\|\nabla \nu\|_{L^{p}} \leq\left(p E_{0}\right)^{\frac{1}{p}}=: C_{10} .
$$

Suppose that $d=2$. An application of the Sobolev inequality (3.4) in Lemma 3.3 indicates that

$$
\begin{aligned}
p^{\frac{1}{p}}\left(\frac{p}{p-1}\right)^{\frac{p-1}{p}} \varepsilon^{\frac{2(p-1)}{p}} s^{\frac{(p-1)}{p}} C_{9}^{-2}\|\nabla \xi\|_{L^{p}}^{2} & \leq p^{\frac{1}{p}}\|\xi\|^{\frac{2}{p}} \cdot\left(\frac{p}{p-1}\right)^{\frac{p-1}{p}} \varepsilon^{\frac{2(p-1)}{p}} s^{\frac{p-1}{p}}\|\Delta \xi\|^{\frac{2(p-1)}{p}} \\
& \leq\|\xi\|^{2}+s \varepsilon^{2}\|\Delta \xi\|^{2},
\end{aligned}
$$

where Young's inequality is applied in the second step. It follows that,

$$
(p-1) s\|\nabla \nu\|_{L^{p}}^{p-2}\|\nabla \xi\|_{L^{p}}^{2} \leq \frac{1}{p}(p-1)^{\frac{2 p-1}{p}} \varepsilon^{\frac{-2(p-1)}{p}} S^{\frac{1}{p}} C_{9}^{2} C_{10}^{p-2}\left(\|\xi\|^{2}+s \varepsilon^{2}\|\Delta \xi\|^{2}\right) .
$$

Substituting (3.10) in (3.9) yields

$$
\left|\delta^{2} E[\nu](\xi, \xi)\right| \leq\left(1+\frac{1}{p}(p-1)^{\frac{2 p-1}{p}} \varepsilon^{\frac{-2(p-1)}{p}} s^{\frac{1}{p}} C_{9}^{2} C_{10}^{p-2}\right)\left(\|\xi\|^{2}+s \varepsilon^{2}\|\Delta \xi\|^{2}\right) .
$$

We conclude that estimate (3.6) is valid by choosing

$$
C_{6}=1+\frac{1}{p}(p-1)^{\frac{2 p-1}{p}} \varepsilon^{\frac{-2(p-1)}{p}} S^{\frac{1}{p}} C_{9}^{2} C_{10}^{p-2} .
$$


Note that both $C_{9}$ and $C_{10}$ are $\varepsilon$ and $s$ independent. Following the similar arguments, for $p \in[2,6), d=3$, we get

$$
C_{6}=1+(p-1)\left(\frac{4 p}{6-p}\right)^{\frac{p-6}{4 p}}\left(\frac{4 p}{5 p-6}\right)^{\frac{6-5 p}{4 p}} s^{\frac{6-p}{4 p}} \varepsilon^{\frac{6-5 p}{2 p}} C_{9}^{2} C_{10}^{p-2} .
$$

For the case $p=6, d=3$, the Sobolev inequality (3.4) degenerates to $\|\nabla \xi\|_{L^{p}} \leq C_{9}\|\Delta \xi\|$, for any $\xi \in H_{\text {per }}^{2}(\Omega)$. Hence, we have

$$
\|\xi\|^{2}+s \varepsilon^{2}\|\Delta \xi\|^{2} \geq s \varepsilon^{2}\|\Delta \xi\|^{2} \geq s \varepsilon^{2} C_{9}^{-2}\|\nabla \xi\|_{L^{p}}^{2}
$$

and

$$
\left|\delta^{2} E[\nu](\xi, \xi)\right| \leq\left(1+(p-1) \varepsilon^{-2} C_{9}^{2} C_{10}^{p-2}\right)\left(\|\xi\|^{2}+\varepsilon^{2}\|\Delta \xi\|^{2}\right) .
$$

Therefore, estimate (3.6) is valid by choosing

$$
C_{6}=1+(p-1) \varepsilon^{-2} C_{9}^{2} C_{10}^{p-2} .
$$

That we can take $C_{8}=C_{5}$ is the result of a simple calculation that we omit for the sake of brevity. The proof is complete.

\section{A Sixth-Order Regularized p-Laplacian Problem}

We now study problem (1.2a) - (1.2b). A weak formulation is given as follows: for $f, g \in L_{\text {per }}^{2}(\Omega)$, find $u \in H_{\mathrm{per}}^{2}(\Omega)$ and $w \in H_{\mathrm{per}}^{1}(\Omega)$ such that

$$
\begin{aligned}
(u, \chi)+(\nabla w, \nabla \chi) & =(g, \chi), \quad \forall \chi \in H_{\mathrm{per}}^{1}(\Omega), \\
s \lambda(u, \xi)+s\left(|\nabla u|^{p-2} \nabla u, \nabla \xi\right)+s \varepsilon^{2}(\Delta u, \Delta \xi)-(w, \xi) & =(f, \xi), \quad \forall \xi \in H_{\mathrm{per}}^{2}(\Omega),
\end{aligned}
$$

where $\lambda \geq 0$, and $\varepsilon \in(0,1]$. This problem is mass-conservative, in the sense that $(u-g, 1)=0$, and $(w-s \lambda g+f, 1)=0$, and it can be recast as a minimization problem with an energy that involves the $\dot{H}_{\text {per }}^{-1}$ norm. In particular, for any $\nu \in \stackrel{\circ}{\text { per }}^{2}(\Omega)$ we define

$$
\begin{aligned}
E[\nu] & =\frac{1}{2}(\nu-g+\bar{g}, \mathrm{~T}[\nu-g+\bar{g}])+\frac{\lambda s}{2}\|\nu+\bar{g}\|^{2}-(\nu, f)+\frac{s}{p}\|\nabla \nu\|_{L^{p}}^{p}+\frac{s \varepsilon^{2}}{2}\|\Delta \nu\|^{2} \\
& =\frac{1}{2}\|\nu-g+\bar{g}\|_{\hat{H}_{\text {per }}^{-1}}^{2}+\frac{\lambda s}{2}\|\nu+\bar{g}\|^{2}-(\nu, f)+\frac{s}{p}\|\nabla \nu\|_{L^{p}}^{p}+\frac{s \varepsilon^{2}}{2}\|\Delta \nu\|^{2},
\end{aligned}
$$

where by $\bar{g}$ we denote the average of $g$ over $\Omega$. Observe that $\nu-g+\bar{g} \in \stackrel{H}{\text { per }}^{-1}$, which is required for this energy to make sense. It is straightforward to show that $E$ satisfies $(E 1)-(E 3)$, with respect to the Hilbert space $H=\stackrel{\circ}{\text { per }}_{2}^{2}(\Omega)$. The first variation of $E$ is given as follows: for any $\xi \in \stackrel{\circ}{\text { per }}^{2}(\Omega)$,

$$
\begin{aligned}
\left.d_{\tau} E[\nu+\tau \xi]\right|_{\tau=0}=\delta E[\nu](\xi)= & (\mathrm{T}[\nu-g+\bar{g}], \xi)+\lambda s(\nu+\bar{g}, \xi)-(f, \xi) \\
& +s\left(|\nabla \nu|^{p-2} \nabla \nu, \nabla \xi\right)+s \varepsilon^{2}(\Delta \nu, \Delta \xi) .
\end{aligned}
$$

The unique minimizer of $E$ - let us call it $u_{\star} \in \stackrel{\circ}{\text { per }}^{2}(\Omega)$ for the moment - satisfies $\delta E\left[u_{\star}\right](\xi)=0$, for all $\xi \in \stackrel{\circ}{H}_{\text {per }}^{2}(\Omega)$. By the definition of the T operator, there is a unique element $w_{\star} \in \stackrel{\circ}{\text { per }}^{1}(\Omega)$ such that

$$
W_{\star}:=-\mathrm{T}\left[u_{\star}-g+\bar{g}\right] .
$$

Therefore, we have, for all $\xi \in \stackrel{\circ}{\text { per }}^{2}(\Omega)$,

$$
s \lambda\left(u_{\star}+\bar{g}, \xi\right)+s\left(\left|\nabla u_{\star}\right|^{p-2} \nabla u_{\star}, \nabla \xi\right)+s \varepsilon^{2}\left(\Delta u_{\star}, \Delta \xi\right)-\left(w_{\star}, \xi\right)=(f, \xi) .
$$

Setting $u:=u_{\star}+\bar{g}$ and $w:=w_{\star}+s \lambda \bar{g}-\bar{f}$ and using the fact that $\xi$ is of zero mean, we have

$$
s \lambda(u, \xi)+s\left(|\nabla u|^{p-2} \nabla u, \nabla \xi\right)+s \varepsilon^{2}(\Delta u, \Delta \xi)-(w, \xi)=(f, \xi), \quad \forall \xi \in \stackrel{\circ}{\mathrm{per}}^{2} .
$$


Using the definition of the T operator again, we conclude that $w_{\star} \in \stackrel{\circ}{\text { per }}_{1}^{1}(\Omega)$ satisfies

$$
\left(\nabla w_{\star}, \nabla \chi\right)=-\left(u_{\star}-g+\bar{g}, \chi\right)
$$

for all $\chi \in \stackrel{\circ}{\text { per }}_{1}^{1}(\Omega)$, which implies that

$$
(\nabla w, \nabla \chi)=-(u-g, \chi) .
$$

It follows that solving (4.1a) - (4.1b) is equivalent to minimizing the coercive, strictly convex energy (4.2), after the appropriate affine change of variables.

The second variation of $E$ is a continuous bilinear operator. Given a fixed $\nu \in \stackrel{\circ}{\text { per }}^{2}(\Omega)$, the action of the

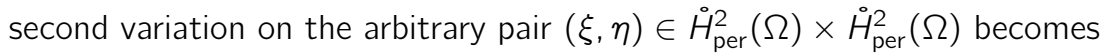

$$
\begin{aligned}
\delta^{2} E[\nu](\xi, \eta)= & (\xi, \mathrm{T}[\eta])+\lambda s(\xi, \eta)+s\left(|\nabla \nu|^{p-2} \nabla \xi, \nabla \eta\right) \\
& +(p-2) s\left(|\nabla \nu|^{p-4} \nabla \nu \cdot \nabla \xi, \nabla \nu \cdot \nabla \eta\right)+s \varepsilon^{2}(\Delta \xi, \Delta \eta) .
\end{aligned}
$$

Similar to the estimate in the fourth-order case (3.3), we have the bound

$$
\begin{aligned}
& \left|\delta^{2} E[\nu](\xi, \eta)\right| \leq\|\xi\|_{\dot{H}_{\mathrm{per}}^{-1}}\|\eta\|_{\stackrel{\leftrightarrow}{\text { per }}^{-1}}+\lambda s\|\xi\| \cdot\|\eta\|+s\|\nabla \nu\|_{L^{p}}^{p-2}\|\nabla \xi\|_{L^{p}}\|\nabla \eta\|_{L^{p}} \\
& +(p-2) s\|\nabla \nu\|_{L^{p}}^{p-2}\|\nabla \xi\|_{L^{p}}\|\nabla \eta\|_{L^{p}}+s \varepsilon^{2}\|\Delta \xi\| \cdot\|\Delta \eta\| \text {. }
\end{aligned}
$$

which implies that

$$
\left|\delta^{2} E[\nu](\xi, \xi)\right| \leq\|\xi\|_{\dot{H}_{\text {per }}^{-1}}^{2}+s \lambda\|\xi\|^{2}+(p-1) s\|\nabla \nu\|_{L^{p}}^{p-2}\|\nabla \xi\|_{L^{p}}^{2}+s \varepsilon^{2}\|\Delta \xi\|^{2},
$$

for all $\nu, \xi \in \stackrel{\circ}{\text { per }}^{2}(\Omega)$.

For the sixth order problem, we define the pre-conditioner $\mathcal{L}: \stackrel{\circ}{\text { per }}^{2}(\Omega) \rightarrow \stackrel{\circ}{\text { per }}^{-2}(\Omega)$ via

$$
\langle\mathcal{L}[\nu], \xi\rangle:=s \lambda(\nu, \xi)+(\nu, \xi)_{\dot{H}_{\mathrm{per}}^{-1}}+s(\nabla \nu, \nabla \xi)+s \varepsilon^{2}(\Delta \nu, \Delta \xi), \quad \forall \xi \in \stackrel{\circ}{\mathrm{per}}^{2}(\Omega) .
$$

This operator satisfies $(L 1)-(L 3)$. To show that it satisfies (L3) - (L6), we need some technical results.

Lemma 4.1. For every $\xi \in \stackrel{\circ}{\text { per }}^{2}(\Omega)$ we have

$$
\|\xi\| \leq\|\xi\|_{\dot{H}_{\text {per }}^{-1}}^{\frac{2}{3}}\|\Delta \xi\|^{\frac{1}{3}}
$$

and

$$
\|\nabla \xi\| \leq\|\xi\|_{\dot{H}_{\text {per }}^{-1}}^{\frac{1}{3}}\|\Delta \xi\|^{\frac{2}{3}}
$$

Proof. Using integration by parts we get

$$
\|\nabla \xi\|^{2}=-(\xi, \Delta \xi) \leq\|\xi\| \cdot\|\Delta \xi\| .
$$

The definition of the $\stackrel{\circ}{\text { per }}^{-1}(\Omega)$ norm implies that

$$
\|\xi\|^{2}=(\xi, \xi) \leq\|\xi\|_{\hat{H}_{\text {per }}^{-1}}\|\nabla \xi\| .
$$

Therefore, a combination of (4.7) and (4.8) leads to

$$
\|\nabla \xi\| \leq\|\xi\|^{\frac{1}{2}} \cdot\|\Delta \xi\|^{\frac{1}{2}} \leq\|\xi\|_{\stackrel{H}{\text { per }}^{-1}}^{\frac{1}{4}}\|\nabla \xi\|^{\frac{1}{4}} \cdot\|\Delta \xi\|^{\frac{1}{2}},
$$

so that

$$
\|\nabla \xi\|^{\frac{3}{4}} \leq\|\xi\|_{\dot{H}_{\text {per }}^{-1}}^{\frac{1}{4}}\|\Delta \xi\|^{\frac{1}{2}} .
$$

which yields the second inequality. The first may be proved in a similar way. 
Similar to before, the Gagliardo-Nirenberg inequality, together with elliptic regularity, yield the following interpolation result.

Lemma 4.2. Suppose that $p \in[2, \infty)$ when $d=2$, and $p \in[2,6]$, if $d=3$. For any $\xi \in \stackrel{\circ}{\mathrm{per}}^{2}(\Omega)$, we have

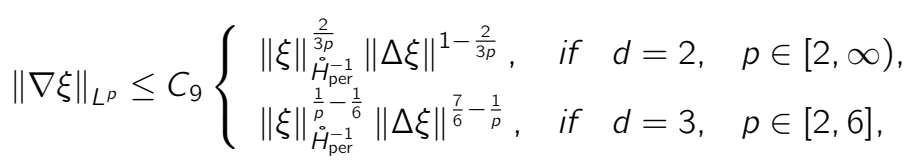

for some $C_{9}=C_{9}(d, p)>0$.

We can now find the coefficients $C_{5}, C_{6}$, and $C_{8}$, which establish properties (L4) - (L6) and therefore guarantee the geometric convergence of the PSD method for the sixth-order case.

Lemma 4.3. For any $\nu, \xi \in \stackrel{\circ}{\mathrm{per}}^{2}(\Omega)$, we have

$$
C_{5}\|\xi-\nu\|_{\mathcal{L}}^{2} \leq(\delta E[\xi]-\delta E[\nu])(\xi-\nu),
$$

where $C_{5}=\min \left(\frac{1}{3}, \varepsilon^{\frac{4}{3}} S^{-\frac{1}{3}}\right)$. Let $E_{0}$ be given such that $B:=\left\{\xi \in \stackrel{\circ}{\mathrm{per}}^{2}(\Omega) \mid E[\xi] \leq E_{0}\right\}$ is non-empty. For any $\nu \in B$ and any $\xi \in \dot{H}_{\text {per }}^{2}(\Omega)$, the following estimate is valid:

$$
\left|\delta^{2} E[\nu](\xi, \xi)\right| \leq C_{6}\|\xi\|_{\mathcal{L}}^{2}
$$

where

$$
C_{6}= \begin{cases}1+(p-1)\left(\frac{3 p}{2}\right)^{-\frac{2}{3 p}}\left(\frac{3 p}{3 p-2}\right)^{\frac{2-3 p}{3 p}} \varepsilon^{\frac{4-6 p}{3 p}} s^{\frac{2}{3 p}} C_{9}^{2} C_{10}^{p-2}, & \text { for } p \in[2, \infty), d=2, \\ 1+(p-1)\left(\frac{6 p}{6-p}\right)^{\frac{p-6}{6 p}}\left(\frac{6 p}{7 p-6}\right)^{\frac{6-7 p}{6 p}} \varepsilon^{\frac{6-7 p}{3 p}} s^{\frac{6-p}{6 p}} C_{9}^{2} C_{10}^{p-2}, & \text { for } p \in[2,6), d=3 \\ 1+(p-1) \varepsilon^{-2} C_{9}^{2} C_{10}^{p-2}, & \text { for } p=6, d=3\end{cases}
$$

and $C_{10}=\left(p E_{0}\right)^{\frac{1}{p}}$. We can take $C_{8}=C_{5}$ to satisfy estimate (2.15) of assumption (L6).

Proof. The proof is similar to that of Lemma 3.4, and we omit it for the sake of brevity. The details may be found in [17].

Remark 4.4. We note that a mixed formulation of the sixth-order regularized p-Laplacian problem - expressed in strong form in (1.2a) - (1.2b) and in weak form in (4.1a) - (4.1b) - in order to preserve the proper variational structure of the problem. Specifically, observe that the $p$-Laplacian term appearing in (1.2b) is the gradient of a convex energy functional. However, if one applies $-\Delta$ to (1.2b), so that the variable $w$ is dropped, a composition of the p-Laplacian and regular Laplacian operators yields a nonlinear term that could not be represented as the gradient of a convex energy. In short, the variational/convexity structure would be lost and the the theoretical convergence could not be justified.

\section{Finite Difference Spatial Discretization in 2D}

\subsection{Notation}

In this subsection we define the discrete spatial difference operators, function space, inner products and norms, following the notation used in $[24,32,35,36,37]$. Let $\Omega=(0, L) \times(0, L)$, with $L>0$. We write $L=m \cdot h$, where $m$ is a positive integer. The parameter $h=\frac{L}{m}$ is called the mesh or grid spacing. We define the following two uniform, infinite grids with grid spacing $h>0$ :

$$
E:=\left\{x_{i+\frac{1}{2}} \mid i \in \mathbb{Z}\right\}, \quad C:=\left\{x_{i} \mid i \in \mathbb{Z}\right\},
$$


where $x_{i}=x(i):=\left(i-\frac{1}{2}\right) \cdot h$. Consider the following 2D discrete periodic function spaces:

$$
\begin{aligned}
& \mathcal{V}_{\text {per }}:=\left\{\nu: E \times E \rightarrow \mathbb{R} \mid \nu_{i+\frac{1}{2}, j+\frac{1}{2}}=\nu_{i+\frac{1}{2}+\alpha m, j+\frac{1}{2}+\beta m}, \forall i, j, \alpha, \beta \in \mathbb{Z}\right\}, \\
& \mathcal{C}_{\text {per }}:=\left\{\nu: C \times C \rightarrow \mathbb{R} \mid \nu_{i, j}=\nu_{i+\alpha m, j+\beta m}, \forall i, j, \alpha, \beta \in \mathbb{Z}\right\}, \\
& \mathcal{E}_{\text {per }}^{\text {ew }}:=\left\{\nu: E \times C \rightarrow \mathbb{R} \mid \nu_{i+\frac{1}{2}, j}=\nu_{i+\frac{1}{2}+\alpha m, j+\beta m}, \forall i, j, \alpha, \beta \in \mathbb{Z}\right\}, \\
& \mathcal{E}_{\text {per }}^{\text {ns }}:=\left\{\nu: C \times E \rightarrow \mathbb{R} \mid \nu_{i, j+\frac{1}{2}}=\nu_{i+\alpha m, j+\frac{1}{2}+\beta m}, \forall i, j, \alpha, \beta \in \mathbb{Z}\right\} .
\end{aligned}
$$

The functions of $\mathcal{V}_{\text {per }}$ are called vertex centered functions; those of $\mathcal{C}_{\text {per }}$ are called cell centered functions. The functions of $\mathcal{E}_{\mathrm{per}}^{\mathrm{ew}}$ are called east-west edge-centered functions, and the functions of $\mathcal{E}_{\text {per }}^{\text {ns }}$ are called north-south edge-centered functions. We also define the mean zero space

$$
\stackrel{\mathcal{C}}{\text { per }}:=\left\{\nu \in \mathcal{C}_{\text {per }} \mid \frac{h^{2}}{|\Omega|} \sum_{i, j=1}^{m} \nu_{i, j}=: \bar{\nu}=0\right\} .
$$

We now define the important difference and average operators on the spaces:

$$
\begin{array}{ll}
A_{x} \nu_{i+\frac{1}{2}, \square}:=\frac{1}{2}\left(\nu_{i+1, \square}+\nu_{i, \square}\right), \quad D_{x} \nu_{i+\frac{1}{2}, \square}:=\frac{1}{h}\left(\nu_{i+1, \square}-\nu_{i, \square}\right), \\
A_{y} \nu_{\square, i+\frac{1}{2}}:=\frac{1}{2}\left(\nu_{\square, i+1}+\nu_{\square, i}\right), \quad D_{y} \nu_{\square, i+\frac{1}{2}}:=\frac{1}{h}\left(\nu_{\square, i+1}-\nu_{\square, i}\right),
\end{array}
$$

with $A_{x}, D_{x}: \mathcal{C}_{\text {per }} \rightarrow \mathcal{E}_{\text {per }}^{\text {ew }}$ if $\square$ is an integer, and $A_{x}, D_{x}: \mathcal{E}_{\text {per }}^{\text {ns }} \rightarrow \mathcal{V}_{\text {per }}$ if $\square$ is a half-integer, with $A_{y}, D_{y}$ : $\mathcal{C}_{\text {per }} \rightarrow \mathcal{E}_{\text {per }}^{\text {ns }}$ if $\square$ is an integer, and $A_{y}, D_{y}: \mathcal{E}_{\text {per }}^{\text {ew }} \rightarrow \mathcal{V}_{\text {per }}$ if $\square$ is a half-integer. Likewise,

$$
\begin{array}{ll}
a_{x} \nu_{i, \square}:=\frac{1}{2}\left(\nu_{i+\frac{1}{2}, \square}+\nu_{i-\frac{1}{2}, \square}\right), & d_{x} \nu_{i, \square}:=\frac{1}{h}\left(\nu_{i+\frac{1}{2}, \square}-\nu_{i-\frac{1}{2}, \square}\right), \\
a_{y} \nu_{\square, j}:=\frac{1}{2}\left(\nu_{\square, j+\frac{1}{2}}+\nu_{\square, j-\frac{1}{2}}\right), & d_{y} \nu_{\square, j}:=\frac{1}{h}\left(\nu_{\square, j+\frac{1}{2}}-\nu_{\square, j-\frac{1}{2}}\right),
\end{array}
$$

with $a_{x}, d_{x}: \mathcal{E}_{\text {per }}^{\mathrm{ew}} \rightarrow \mathcal{C}_{\text {per }}$ if $\square$ is an integer, and $a_{x}, d_{x}: \mathcal{V}_{\text {per }} \rightarrow \mathcal{E}_{\text {per }}^{\text {ns }}$ if $\square$ is a half-integer; and with $a_{y}, d_{y}$ : $\mathcal{E}_{\text {per }}^{\text {ns }} \rightarrow \mathcal{C}_{\text {per }}$ if $\square$ is an integer, and $a_{y}, d_{y}: \mathcal{V}_{\text {per }} \rightarrow \mathcal{E}_{\text {per }}^{\text {ew }}$ if $\square$ is a half-integer.

Define the 2D center-to-vertex derivatives $\mathfrak{D}_{x}, \mathfrak{D}_{y}: \mathcal{C}_{\text {per }} \rightarrow \mathcal{V}_{\text {per }}$ component-wise as

$$
\begin{aligned}
\mathfrak{D}_{x} \nu_{i+\frac{1}{2}, j+\frac{1}{2}} & :=A_{y}\left(D_{x} \nu\right)_{i+\frac{1}{2}, j+\frac{1}{2}}=D_{x}\left(A_{y} \nu\right)_{i+\frac{1}{2}, j+\frac{1}{2}} \\
& =\frac{1}{2 h}\left(\nu_{i+1, j+1}-\nu_{i, j+1}+\nu_{i+1, j}-\nu_{i, j}\right) . \\
\mathfrak{D}_{y} \nu_{i+\frac{1}{2}, j+\frac{1}{2}} & :=A_{x}\left(D_{y} \nu\right)_{i+\frac{1}{2}, j+\frac{1}{2}}=D_{y}\left(A_{x} \nu\right)_{i+\frac{1}{2}, j+\frac{1}{2}} \\
& =\frac{1}{2 h}\left(\nu_{i+1, j+1}-\nu_{i+1, j}+\nu_{i, j+1}-\nu_{i, j}\right) .
\end{aligned}
$$

The utility of these definitions is that the differences $\mathfrak{D}_{x}$ and $\mathfrak{D}_{y}$ are collocated on the grid, unlike the case for $D_{x}, D_{y}$. Define the $2 \mathrm{D}$ vertex-to-center derivatives $\mathfrak{d}_{x}, \mathfrak{d}_{y}: \mathcal{V}_{\text {per }} \rightarrow \mathcal{C}_{\text {per }}$ component-wise as

$$
\begin{aligned}
\mathfrak{d}_{x} \nu_{i, j} & :=a_{y}\left(d_{x} \nu\right)_{i, j}=d_{x}\left(a_{y} \nu\right)_{i, j} \\
& =\frac{1}{2 h}\left(\nu_{i+\frac{1}{2}, j+\frac{1}{2}}-\nu_{i-\frac{1}{2}, j+\frac{1}{2}}+\nu_{i+\frac{1}{2}, j-\frac{1}{2}}-\nu_{i-\frac{1}{2}, j-\frac{1}{2}}\right), \\
\mathfrak{d}_{y} \nu_{i, j} & :=a_{x}\left(d_{y} \nu\right)_{i, j}=d_{y}\left(a_{x} \nu\right)_{i, j} \\
& =\frac{1}{2 h}\left(\nu_{i+\frac{1}{2}, j+\frac{1}{2}}-\nu_{i+\frac{1}{2}, j-\frac{1}{2}}+\nu_{i-\frac{1}{2}, j+\frac{1}{2}}-\nu_{i-\frac{1}{2}, j-\frac{1}{2}}\right) .
\end{aligned}
$$

Now the discrete gradient operator, $\nabla_{h}^{v}: \mathcal{C}_{\text {per }} \rightarrow \mathcal{V}_{\text {per }} \times \mathcal{V}_{\text {per }}$, is defined as

$$
\nabla_{h}^{V} \nu_{i+\frac{1}{2}, j+\frac{1}{2}}:=\left(\mathfrak{D}_{x} \nu_{i+\frac{1}{2}, j+\frac{1}{2}}, \mathfrak{D}_{y} \nu_{i+\frac{1}{2}, j+\frac{1}{2}}\right) .
$$


The standard 2D discrete Laplacian, $\Delta_{h}: \mathcal{C}_{\text {per }} \rightarrow \mathcal{C}_{\text {per, }}$, is given by

$$
\Delta_{h} \nu_{i, j}:=d_{x}\left(D_{x} \nu\right)_{i, j}+d_{y}\left(D_{y} \nu\right)_{i, j}=\frac{1}{h^{2}}\left(\nu_{i+1, j}+\nu_{i-1, j}+\nu_{i, j+1}+\nu_{i, j-1}-4 \nu_{i, j}\right) .
$$

The $2 \mathrm{D}$ vertex-to-center average, $\mathcal{A}: \mathcal{V}_{\text {per }} \rightarrow \mathcal{C}_{\text {per }}$, is defined to be

$$
\mathcal{A} \nu_{i, j}:=\frac{1}{4}\left(\nu_{i+1, j}+\nu_{i-1, j}+\nu_{i, j+1}+\nu_{i, j-1}\right) .
$$

The 2D skew Laplacian, $\Delta_{h}^{v}: \mathcal{C}_{\text {per }} \rightarrow \mathcal{C}_{\text {per }}$, is defined as

$$
\begin{aligned}
\Delta_{h}^{v} \nu_{i, j} & =\mathfrak{d}_{x}\left(\mathfrak{D}_{x} \nu\right)_{i, j}+\mathfrak{d}_{y}\left(\mathfrak{D}_{y} \nu\right)_{i, j} \\
& =\frac{1}{2 h^{2}}\left(\nu_{i+1, j+1}+\nu_{i-1, j+1}+\nu_{i+1, j-1}+\nu_{i-1, j-1}-4 \nu_{i, j}\right) .
\end{aligned}
$$

The $2 \mathrm{D}$ discrete $\mathrm{p}$-Laplacian operator is defined as

$$
\nabla_{h}^{v} \cdot\left(\left|\nabla_{h}^{v} \nu\right|^{p-2} \nabla_{h}^{v} \nu\right)_{i j}:=\mathfrak{d}_{x}\left(r \mathfrak{D}_{x} \nu\right)_{i, j}+\mathfrak{d}_{y}\left(r \mathfrak{D}_{y} \nu\right)_{i, j}
$$

with

$$
r_{i+\frac{1}{2}, j+\frac{1}{2}}:=\left[\left(\mathfrak{D}_{x} u\right)_{i+\frac{1}{2}, j+\frac{1}{2}}^{2}+\left(\mathfrak{D}_{y} u\right)_{i+\frac{1}{2}, j+\frac{1}{2}}^{2}\right]^{\frac{p-2}{2}} .
$$

Clearly, for $p=2, \Delta_{h}^{v} \nu=\nabla_{h}^{v} \cdot\left(\left|\nabla_{h}^{v} \nu\right|^{p-2} \nabla_{h}^{v} \nu\right)$.

Now we are ready to define the following grid inner products:

$$
\begin{aligned}
(\nu, \xi)_{2} & :=h^{2} \sum_{i=1}^{m} \sum_{j=1}^{n} \nu_{i, j} \psi_{i, j}, \quad \nu, \xi \in \mathcal{C}_{\mathrm{per}}, \\
\langle\nu, \xi\rangle & :=(\mathcal{A}(\nu \xi), 1)_{2}, \quad \nu, \xi \in \mathcal{V}_{\mathrm{per}}, \\
{[\nu, \xi]_{\mathrm{ew}} } & :=\left(A_{x}(\nu \xi), 1\right)_{2}, \quad \nu, \xi \in \mathcal{E}_{\mathrm{per}}^{\mathrm{ew}}, \\
{[\nu, \xi]_{\mathrm{ns}} } & :=\left(A_{y}(\nu \xi), 1\right)_{2}, \quad \nu, \xi \in \mathcal{E}_{\mathrm{per}}^{\mathrm{ns}} .
\end{aligned}
$$

Suppose that $\zeta \in \dot{\mathcal{C}}_{\text {per }}$, then there is a unique solution $\mathrm{T}_{h}[\zeta] \in \dot{\mathcal{C}}_{\text {per }}$ such that $-\Delta_{h} \mathrm{~T}_{h}[\zeta]=\zeta$. We often write, in this case, $\mathrm{T}_{h}[\zeta]=-\Delta_{h}^{-1} \zeta$. The discrete analogue of the $\dot{H}_{\mathrm{per}}^{-1}$ inner product is defined as

$$
(\zeta, \xi)_{-1}:=\left(\zeta, \mathbf{T}_{h}[\xi]\right)_{2}=\left(\mathrm{T}_{h}[\zeta], \xi\right)_{2}, \quad \zeta, \xi \in \dot{\mathcal{C}}_{\text {per }}
$$

where summation-by-parts $[32,37]$ guarantees the symmetry and the second equality.

We now define the following norms for cell-centered functions. If $\nu \in \dot{\mathcal{C}}_{\text {per }}$, then $\|\nu\|_{-1}^{2}=(\nu, \nu)_{-1}$. If $\nu \in \mathcal{C}_{\text {per }}$, then $\|\nu\|_{2}^{2}:=(\nu, \nu)_{2} ;\|\nu\|_{p}^{p}:=\left(|\nu|^{p}, 1\right)_{2}(1 \leq p<\infty)$, and $\|\nu\|_{\infty}:=\max _{\substack{1 \leq i \leq m \\ 1 \leq \leq \leq n}}\left|\nu_{i, j}\right|$. Similarly, we define the gradient norms: for $\nu \in \mathcal{C}_{\text {per }}$,

$$
\left\|\nabla_{h}^{v} \nu\right\|_{p}^{p}:=\left\langle\left|\nabla_{h}^{v} \nu\right|^{p}, 1\right\rangle, \quad\left|\nabla_{h}^{v} \nu\right|^{p}:=\left[\left(\mathfrak{D}_{x} \nu\right)^{2}+\left(\mathfrak{D}_{y} \nu\right)^{2}\right]^{\frac{p}{2}}=\left[\nabla_{h}^{v} \nu \cdot \nabla_{h}^{v} \nu\right]^{\frac{p}{2}} \in \mathcal{V}_{\text {per }}, \quad 2 \leq p<\infty,
$$

and

$$
\left\|\nabla_{h} \nu\right\|_{2}^{2}:=\left[D_{x} \nu, D_{x} \nu\right]_{\mathrm{ew}}+\left[D_{y} \nu, D_{y} \nu\right]_{\mathrm{ns}} .
$$

\subsection{Discrete Sobolev Inequalities}

Lemma 5.1. Suppose that $p \in[2, \infty), d=2$, we have

$$
\left\|\nabla_{h}^{v} \xi\right\|_{p} \leq C_{9}\left\{\begin{array}{lll}
\|\xi\|_{2}^{\frac{1}{p}} \cdot\left\|\Delta_{h} \xi\right\|_{2}^{\frac{p-1}{p}}, & \forall & \xi \in \mathcal{C}_{\mathrm{per}}, \\
\|\xi\|_{-1}^{\frac{2}{3 p}} \cdot\left\|\Delta_{h} \xi\right\|_{2}^{1-\frac{2}{3 p}}, & \forall & \xi \in \mathcal{C}_{\mathrm{per}},
\end{array}\right.
$$

for some $C_{9}=C_{9}(p)>0$. 
Proof. The proof for $p=4, d=2$ can be found in [17].

Remark 5.2. Though we have focused on the case $d=2$ in this section, we can also define our operators and norms, in particular $\nabla_{h}^{v} \xi$ and $\left\|\nabla_{h}^{v} \xi\right\|_{p}$, in three space dimensions. Then for $p \in[2,6]$, we expect

$$
\left\|\nabla_{h}^{V} \xi\right\|_{p} \leq C_{9}\left\{\begin{array}{lll}
\|\xi\|_{2}^{\frac{3}{2 p}-\frac{1}{4}}\left\|\Delta_{h} \xi\right\|_{2}^{\frac{5}{4}-\frac{3}{2 p}}, & \forall & \xi \in \mathcal{C}_{\text {per }}, \\
\|\xi\|_{-1}^{\frac{1}{p}-\frac{1}{6}}\left\|\Delta_{h} \xi\right\|_{2}^{\frac{7}{6}-\frac{1}{p}}, & \forall & \xi \in \dot{\mathcal{C}}_{\mathrm{per}},
\end{array}\right.
$$

for some $C_{9}=C_{9}(d=3, p)>0$.

\subsection{Convergence for the Discretized Fourth-Order Problem}

The discrete version of (1.1) can be expressed as follows: given $f \in \mathcal{C}_{\text {per }}$, find $u \in \mathcal{C}_{\text {per }}$ such that

$$
u-s \nabla_{h}^{v} \cdot\left(\left|\nabla_{h}^{v} u\right|^{p-2} \nabla_{h}^{v} u\right)+s \varepsilon^{2} \Delta_{h}^{2} u=f .
$$

This represents a second-order approximation of the solution of (1.1). As in the space continuous case, we formulate an equivalent minimization problem. Using the definitions from subsection 5.1, we have the following discrete energy: given $f \in \mathcal{C}_{\text {per }}$, for any $\nu \in \mathcal{C}_{\text {per }}$, define

$$
E_{h}[\nu]:=\frac{1}{2}\|\nu-f\|_{2}^{2}+\frac{s}{p}\left\|\nabla_{h}^{v} \nu\right\|_{p}^{p}+\frac{s \varepsilon^{2}}{2}\left\|\Delta_{h} \nu\right\|_{2}^{2} .
$$

This (discrete) energy satisfies (E1) - (E3). The discrete variational derivative at $\nu \in \mathcal{C}_{\text {per }}$ is

$$
\begin{aligned}
\delta E_{h}[\nu](\xi) & :=\left.d_{\tau} E_{h}[\nu+\tau \xi]\right|_{\tau=0} \\
& =(\nu-f, \xi)_{2}+s\left\langle\left|\nabla_{h}^{v} \nu\right|^{p-2} \mathfrak{D}_{x} \nu, \mathfrak{D}_{x} \xi\right\rangle+s\left\langle\left|\nabla_{h}^{v} \nu\right|^{p-2} \mathfrak{D}_{y} \nu, \mathfrak{D}_{y} \xi\right\rangle+s \varepsilon^{2}\left(\Delta_{h} \nu, \Delta_{h} \xi\right)_{2} \\
& =(\nu-f, \xi)_{2}+s\left\langle\left|\nabla_{h}^{v} \nu\right|^{p-2} \nabla_{h}^{v} \nu, \nabla_{h}^{v} \xi\right\rangle+s \varepsilon^{2}\left(\Delta_{h} \nu, \Delta_{h} \xi\right)_{2} \\
& =\left(\nu-f-s \nabla_{h}^{v} \cdot\left(\left|\nabla_{h}^{v} \nu\right|^{p-2} \nabla_{h}^{v} \nu\right)+s \varepsilon^{2} \Delta_{h}^{2} \nu, \xi\right)_{2},
\end{aligned}
$$

for all $\xi \in \mathcal{C}_{\text {per }}$, where we have used summation-by-parts $[32,37]$ to obtain the last equality. Given a fixed $\nu \in \mathcal{C}_{\text {per }}$, the action of the second variation on the arbitrary pair $(\xi, \eta) \in \mathcal{C}_{\text {per }} \times \mathcal{C}_{\text {per }}$ is given by

$$
\begin{aligned}
\delta^{2} E_{h}[\nu](\xi, \eta)= & (\xi, \eta)_{2}+s\left\langle\left|\nabla_{h}^{v} \nu\right|^{p-2} \nabla_{h}^{v} \xi, \nabla_{h}^{v} \eta\right\rangle \\
& +(p-2) s\left\langle\left|\nabla_{h}^{v} \nu\right|^{p-4} \nabla_{h}^{v} \nu \cdot \nabla_{h}^{v} \xi, \nabla_{h}^{v} \nu \cdot \nabla_{h}^{v} \eta\right\rangle+s \varepsilon^{2}\left(\Delta_{h} \xi, \Delta_{h} \eta\right)_{2} .
\end{aligned}
$$

We have the bound:

$$
\begin{aligned}
\left|\delta^{2} E_{h}[\nu](\xi, \eta)\right| \leq & \|\xi\|_{2}\|\eta\|_{2}+s\left\|\nabla_{h}^{v} \nu\right\|_{p}^{p-2}\left\|\nabla_{h}^{v} \xi\right\|_{p}\left\|\nabla_{h}^{v} \eta\right\|_{p} \\
& +(p-2) s\left\|\nabla_{h}^{v} \nu\right\|_{p}^{p-2}\left\|\nabla_{h}^{v} \xi\right\|_{p}\left\|\nabla_{h}^{v} \eta\right\|_{p}+s \varepsilon^{2}\left\|\Delta_{h} \xi\right\|_{2}\left\|\Delta_{h} \eta\right\|_{2} .
\end{aligned}
$$

For this problem, we define the pre-conditioner via

$$
\begin{aligned}
(\nu, \xi)_{\mathcal{L}_{h}}=\mathcal{L}_{h}[\nu](\xi): & =(\nu, \xi)_{2}+s\left[D_{x} \nu, D_{x} \xi\right]_{\mathrm{ew}}+s\left[D_{y} \nu, D_{y} \xi\right]_{\mathrm{ns}}+s \varepsilon^{2}\left(\Delta_{h} \nu, \Delta_{h} \xi\right)_{2} \\
& =\left(\nu-s \Delta_{h} \nu+s \varepsilon^{2} \Delta_{h}^{2} \nu, \xi\right)_{2},
\end{aligned}
$$

for all $\nu, \xi \in \mathcal{C}_{\text {per }}$, where we have used summation-by-parts to establish the second equality. In other words,

$$
\mathcal{L}_{h}[\nu]=\nu-s \Delta_{h} \nu+s \varepsilon^{2} \Delta_{h}^{2} \nu .
$$

One will notice the similarity of the pre-conditioner with the nonlinear operator in (5.1). The induced norm is

$$
\|\nu\|_{\mathcal{L}_{h}}^{2}:=(\nu, \nu)_{\mathcal{L}_{h}}=\|\nu\|_{2}^{2}+s\left\|\nabla_{h} \nu\right\|_{2}^{2}+s \varepsilon^{2}\left\|\Delta_{h} \nu\right\|_{2},
$$

defined for every $\nu \in \mathcal{C}_{\text {per }}$.

Mimicking the proofs in the continuous case, using summation-by-parts in place of integration-by-parts, and Lemma 5.1, we get the following result, whose proof is omitted: 
Lemma 5.3. For any $\nu, \xi \in \mathcal{C}_{\text {per }}$,

$$
C_{5}\|\xi-\nu\|_{\mathcal{L}_{h}}^{2} \leq\left(\delta E_{h}[\xi]-\delta E_{h}[\nu]\right)(\xi-\nu)
$$

where $C_{5}=\min \left(\frac{1}{2}, \varepsilon s^{-\frac{1}{2}}\right)$. Let $E_{0}$ be given, such that $B:=\left\{\nu \in \mathcal{C}_{\text {per }} \mid E_{h}[\nu] \leq E_{0}\right\}$ is non-empty. For any $\nu \in B$ and any $\xi \in \mathcal{C}_{\text {per }}$, we have

$$
\left|\delta^{2} E_{h}[\nu](\xi, \xi)\right| \leq C_{6}\|\xi\|_{\mathcal{L}_{h}}^{2}
$$

where

$$
C_{6}=1+\frac{1}{p}(p-1)^{\frac{2 p-1}{p}} \varepsilon^{\frac{-2(p-1)}{p}} S^{\frac{1}{p}} C_{9}^{2} C_{10}^{p-2},
$$

and $C_{10}=\left(p E_{0}\right)^{\frac{1}{p}}$. We can take $C_{8}=C_{5}$ to satisfy estimate (2.15) of assumption (L6).

\subsection{Convergence for the Discretized Sixth-Order Problem}

The (second-order accurate) discrete version of $(1.2 \mathrm{a})-(1.2 \mathrm{~b})$ can be expressed as follows: given $f, g \in \mathcal{C}_{\text {per }}$, find $u, w \in \mathcal{C}_{\text {per }}$ such that

$$
\begin{aligned}
u-\Delta_{h} w & =g, \\
s \lambda u-s \nabla_{h}^{v} \cdot\left(\left|\nabla_{h}^{v} u\right|^{p-2} \nabla_{h}^{v} u\right)+s \varepsilon^{2} \Delta_{h}^{2} u-w & =f .
\end{aligned}
$$

As before, it is convenient to switch to the mean-zero version: find $u_{\star}, w_{\star} \in \dot{\mathcal{C}}_{\text {per }}$ such that

$$
\begin{aligned}
u_{\star}-\Delta_{h} w_{\star} & =g-\bar{g}, \\
s \lambda u_{\star}-s \nabla_{h}^{v} \cdot\left(\left|\nabla_{h}^{v} u_{\star}\right|^{p-2} \nabla_{h}^{v} u_{\star}\right)+s \varepsilon^{2} \Delta_{h}^{2} u_{\star}-w_{\star} & =f-\bar{f} .
\end{aligned}
$$

Similar to fourth-order regularized p-Laplacian problem, we define the following discrete energy: for every $\nu \in \stackrel{\check{\mathcal{C}}}{\text { per }}$

$$
E_{h}[\nu]:=\frac{1}{2}\|\nu-g+\bar{g}\|_{-1}^{2}+\frac{\lambda s}{2}\|\nu+\bar{g}\|_{2}^{2}-(\nu, f)+\frac{s}{p}\left\|\nabla_{h}^{v} \nu\right\|_{p}^{p}+\frac{s \varepsilon^{2}}{2}\left\|\Delta_{h} \nu\right\|_{2}^{2} .
$$

For the discrete sixth order problem, we define a linear operator $\mathcal{L}_{h}: \stackrel{\circ}{\mathcal{C}}$ per $\rightarrow \dot{\mathcal{C}}_{\text {per }}$ via

$$
\begin{aligned}
(\nu, \xi)_{\mathcal{L}_{h}}=\mathcal{L}_{h}[\nu](\xi): & =s \lambda(\nu, \xi)_{2}+(\nu, \xi)_{-1}+s\left[D_{x} \nu, D_{x} \xi\right]_{\mathrm{ew}}+s\left[D_{y} \nu, D_{y} \xi\right]_{\mathrm{ns}}+s \varepsilon^{2}\left(\Delta_{h} \nu, \Delta_{h} \xi\right)_{2} \\
& =\left(s \lambda \nu-s \Delta_{h} \nu+s \varepsilon^{2} \Delta_{h}^{2} \nu-\mathrm{T}_{h}[-\nu], \xi\right)_{2},
\end{aligned}
$$

where the second equality may be seen using summation-by-parts $[32,37]$. This operator satisfies (L1) - (L3), and the next result, which we give without proof for the sake of brevity, shows that (L4) - (L6) are satisfied as well.

Lemma 5.4. For any $\nu, \xi \in \mathcal{\mathcal { C }}_{\text {per }}$, the following inequality is valid

$$
C_{5}\|\xi-\nu\|_{\mathcal{L}_{h}}^{2} \leq\left(\delta E_{h}[\xi]-\delta E_{h}[\nu]\right)(\xi-\nu),
$$

where $C_{5}=\min \left(\frac{1}{3}, \varepsilon^{\frac{4}{3}} S^{-\frac{1}{3}}\right)$. Let $E_{0}$ be given such that $B:=\left\{\xi \in \stackrel{\mathcal{C}}{\text { per }} \mid E_{h}[\xi] \leq E_{0}\right\}$ is non-empty. For any $\nu \in B$, we have

$$
\left|\delta^{2} E_{h}[\nu](\xi, \xi)\right| \leq C_{6}\|\xi\|_{\mathcal{L}_{h}}^{2}
$$

for all $\xi \in \dot{\mathcal{C}}_{\text {per }}$, where

$$
C_{6}=1+(p-1)\left(\frac{3 p}{2}\right)^{-\frac{2}{3 p}}\left(\frac{3 p}{3 p-2}\right)^{\frac{2-3 p}{3 p}} \varepsilon^{\frac{4-6 p}{3 p}} s^{\frac{2}{3 p}} C_{9}^{2} C_{10}^{p-2},
$$

and $C_{10}=\left(p E_{h, 0}\right)^{\frac{1}{p}}$. We can take $C_{8}=C_{5}$ to satisfy estimate (2.15) of assumption (L6). 


\section{Numerical Experiments}

In this section we perform some numerical experiments to support the theoretical results. The finite difference search direction equations and Poisson equations are solved efficiently using the Fast Fourier Transform (FFT). We would like to point out that the Fourier pseudo-spectral method can be used to discretize space, and, once again, one can utilize the FFT for the inversion of the linear systems. For descriptions of the pseudo-spectral methods, see, for example, $[4,6,21]$.

\subsection{Thin Film Epitaxy Model with Slope Selection}

In this section we recall the convex decomposition numerical scheme in [35] for the thin film epitaxy model with slope selection. Suppose that $\Omega \subset \mathbb{R}^{2}$ is a rectangular domain. The energy of an epitaxial thin film is given by

$$
\mathcal{E}[u]=\int_{\Omega}\left\{\frac{1}{p}|\nabla u|^{p}-\frac{1}{2}|\nabla u|^{2}+\frac{\varepsilon^{2}}{2}|\Delta u|^{2}\right\} d \mathbf{x}, \quad \forall u \in H_{\text {per }}^{2}(\Omega),
$$

where, $p \geq 4$ is even, $u: \Omega \rightarrow \mathbb{R}$ is the height film, and $\varepsilon$ is a constant. The $L^{2}$ gradient flow is

$$
\partial_{t} u=-w, \quad w:=\delta \mathcal{E}=-\nabla \cdot\left(|\nabla u|^{p-2} \nabla u\right)+\Delta u+\varepsilon^{2} \Delta^{2} u
$$

and $w$ is called the chemical potential. The model predicts the emergence of a faceted thin film, whose facets have slopes of magnitude approximately one, that coarsens over time. The fully-implicit convex decomposition scheme in 2D [35] can be written in operator format as $\mathcal{N}_{h}\left[u^{n+1}\right]=f$, where

$$
\mathcal{N}_{h}[\nu]:=\nu-s \nabla_{h}^{v} \cdot\left(\left|\nabla_{h}^{v} \nu\right|^{p-2} \nabla_{h}^{v} \nu\right)+\varepsilon^{2} s \Delta_{h}^{2} \nu, \quad f=u^{n}-s \Delta_{h}^{v} u^{n},
$$

and $s>0$ is the time step. Hence, the scheme can be reformulated as the fourth-order problem (5.1) with $f=u^{n}-s \Delta_{h}^{v} u^{n}$ and $p \geq 4$ and even.

In way of summary, to solve $\mathcal{N}_{h}[u]=f$, suppose that iterate $u^{k} \in \mathcal{C}_{\text {per }}$ is given. (Note that $k$ is the PSD solver iteration index, not the time step index, the latter of which we usually denote by $n$.) We first compute the search direction $d^{k} \in \mathcal{C}_{\text {per }}$ via (2.3):

$$
\begin{aligned}
\mathcal{L}_{h}\left[d^{k}\right]=d^{k}-s \Delta_{h} d^{k}+s \varepsilon^{2} \Delta_{h}^{2} d^{k} & =-\delta E_{h}\left[u^{k}\right] \\
& =-\left(u^{k}-f-s \nabla_{h}^{v} \cdot\left(\left|\nabla_{h}^{v} u^{k}\right|^{p-2} \nabla_{h}^{v} u^{k}\right)+s \varepsilon^{2} \Delta_{h}^{2} u^{k}\right) \\
& =f-\mathcal{N}_{h}\left[u^{k}\right],
\end{aligned}
$$

where $E_{h}$ is as defined in (5.2). This equation is efficiently solved using FFT. Once $d^{k}$ is found, we perform a line-search according to (2.6): find $\alpha_{k} \in \mathbb{R}$ such that $q\left(\alpha_{k}\right)=0$, where

$$
\begin{aligned}
q(\alpha) & :=\delta E_{h}\left[u^{k}+\alpha d^{k}\right]\left(d^{k}\right) \\
& =\left(u^{k}+\alpha d^{k}-f-s \nabla_{h}^{v} \cdot\left(\left|\nabla_{h}^{v}\left(u^{k}+\alpha d^{k}\right)\right|^{p-2} \nabla_{h}^{v}\left(u^{k}+\alpha d^{k}\right)\right)+s \varepsilon^{2} \Delta_{h}^{2}\left(u^{k}+\alpha d^{k}\right), d^{k}\right)_{2} \\
& =\left(\mathcal{N}_{h}\left[u^{k}+\alpha d^{k}\right]-f, d^{k}\right)_{2} .
\end{aligned}
$$

The approximation sequence is then updated via $u^{k+1}=u^{k}+\alpha_{k} d^{k}$. When $p=4(p=6)$, a short calculation shows that $q$ is a cubic (quintic) polynomial whose coefficients can be easily obtained. Moreover, the theory predicts that there is a unique global root for $q$.

\subsubsection{Convergence and complexity of the PSD solver}

In this subsection we demonstrate the accuracy and efficiency of the PSD solver by using the epitaxial thin film model with slope selection. We present the results of some convergence tests and perform some sample computations to demonstrate the convergence and near optimal complexity with respect to the grid size $h$. 
Table 1: Errors, convergence rates, average iteration numbers and average CPU time for each time step. Parameters are given in the text, and the initial data are defined in (6.3). The refinement path is $s=0.1 h^{2}$.

\begin{tabular}{cccccccccc}
\hline \multicolumn{1}{c}{$p=4$} & \multicolumn{9}{c}{$p=6$} \\
\hline$h_{c}$ & $h_{f}$ & $\left\|\delta_{u}\right\|_{2}$ & Rate & $\#_{\text {iter }}$ & $T_{c p u}\left(h_{f}\right)$ & $\left\|\delta_{u}\right\|_{2}$ & Rate & $\#_{\text {iter }}$ & $T_{c p u}\left(h_{f}\right)$ \\
\hline$\frac{3.2}{16}$ & $\frac{3.2}{32}$ & $6.2192 \times 10^{-3}$ & - & 4 & 0.0007 & $9.3074 \times 10^{-3}$ & - & 5 & 0.0009 \\
$\frac{3.2}{32}$ & $\frac{3.2}{64}$ & $1.2685 \times 10^{-3}$ & 2.29 & 2 & 0.0024 & $1.6392 \times 10^{-3}$ & 2.51 & 3 & 0.0032 \\
$\frac{3.2}{64}$ & $\frac{3.2}{128}$ & $2.6046 \times 10^{-4}$ & 2.28 & 2 & 0.0114 & $2.9046 \times 10^{-4}$ & 2.50 & 2 & 0.0141 \\
$\frac{3.2}{128}$ & $\frac{3.2}{256}$ & $5.9639 \times 10^{-5}$ & 2.13 & 2 & 0.0475 & $6.5325 \times 10^{-5}$ & 2.15 & 2 & 0.0616 \\
$\frac{3.2}{256}$ & $\frac{3.2}{512}$ & $1.4526 \times 10^{-5}$ & 2.04 & 2 & 0.3560 & $1.5886 \times 10^{-5}$ & 2.04 & 2 & 0.4636 \\
\hline
\end{tabular}

To simultaneously demonstrate the spatial accuracy and the efficiency of the solver, we perform a typical time-space convergence test for the fully discrete scheme (6.2) for the slope selection model. As in [32, 35], we perform the Cauchy-type convergence test using the following periodic initial data [32]:

$$
\begin{aligned}
u(x, y, 0)= & 0.1 \sin ^{2}\left(\frac{2 \pi x}{L}\right) \cdot \sin \left(\frac{4 \pi(y-1.4)}{L}\right) \\
& -0.1 \cos \left(\frac{2 \pi(x-2.0)}{L}\right) \cdot \sin \left(\frac{2 \pi y}{L}\right),
\end{aligned}
$$

where $\Omega=(0,3.2)^{2}$. In this test, we compute the Cauchy difference, $\delta_{u}:=u_{h_{f}}(T)-\mathcal{I}_{c}^{f}\left(u_{h_{c}}(T)\right)$, where $h_{c}=2 h_{f}$, and $\mathcal{I}_{c}^{f}$ is a bilinear interpolation operator that maps the coarse grid approximation $u_{h_{c}}$ onto the fine grid. We take a quadratic refinement path, i.e., $s=h^{2} / 10$, to equalize the spatial and temporal error contributions. At the final time, $T=0.32$, we expect the global error to be $\mathcal{O}(s)+\mathcal{O}\left(h^{2}\right)=\mathcal{O}\left(h^{2}\right)$ in the discrete $\|\cdot\|_{2}$ and $\|\cdot\|_{\infty}$ norms, as $h, s \rightarrow 0$. The other parameter is given by $\varepsilon=0.1$. The norms of Cauchy difference, the convergence rates, average iteration number and average CPU time can be found on Table 1. Second-order convergence is observed. At the same time, the average iteration count for the solver remains at around 2. Since we are using a quadratic refinement path, increasing the grid size by a factor of two (decreasing the grid spacing by 2) means increasing the number of time-space degrees of freedom by a factor of 16 . But the CPU time increases at a much slower rate. The complexity can be offset, of course, by the fact the starting guesses for the solver at each independent time level are better for smaller time step sizes.

To more directly investigate the complexity of the PSD solver we perform another series of tests to determine the dependences of the convergence rates on $\varepsilon, h, s$, and $p$, in particular. Consider the following spatially periodic function parametrized by $s$ :

$$
\tilde{u}(x, y, s)=\frac{1}{2 \pi} \sin (2 \pi x) \cos (2 \pi y) \cos (s) .
$$

First we calculate $f:=\mathcal{N}_{h}\left[\mathcal{I}_{h}(\tilde{u}(\cdot, \cdot, s))\right] \in \mathcal{C}_{\text {per }}$, where $\mathcal{I}_{h}: C_{\text {per }}^{0}(\Omega) \rightarrow \mathcal{C}_{\text {per }}$ is the canonical grid projection operator. Then we compute the sequence $\left\{u^{k}\right\}_{k=0}^{\infty}$ via the PSD algorithm, with the initialization

$$
u_{i, j}^{0}=\tilde{u}\left(p_{i}, p_{j}, 0\right)+s^{2} \sin \left(4 \pi p_{i}\right) \sin \left(6 \pi p_{j}\right),
$$

hence $u^{k} \rightarrow \mathcal{I}_{h}(\tilde{u}(\cdot, \cdot, s))$, as $k \rightarrow \infty$. Define $\gamma_{k}:=\left\|u^{k}-\mathcal{I}_{h}(\tilde{u}(\cdot, \cdot, s))\right\|_{\infty}$. We stop the PSD algorithm when $\gamma_{k} \leq \tau:=1 \times 10^{-8}$.

In Figure 2 we plot $\gamma_{k}$ versus $k$, on a semi-log scale, for various choices of $h, \varepsilon, s$ and $p$. In Figure 2(a) $p=4$, $s=0.01$ and $\varepsilon=0.03$; in Figure 2(b) $p=4, s=0.01$ and $h=1 / 512$; in Figure 2(c) $p=4, h=1 / 512$ and $\varepsilon=0.03$; in Figure 2(d): $h=1 / 512, s=0.01$ and $\varepsilon=0.03$. As can be seen in Figure 2(a), the convergence rate (as gleaned from the error reduction) is nearly uniform and nearly independent of $h$. Figures 2 (b) and (c) indicate that more PSD iterations are required for smaller values of $\varepsilon$ and larger values of $s$, respectively. Figure 2(d) shows that the number of PSD iterations increases with the value of $p$. These general trends are expected form the theory. 


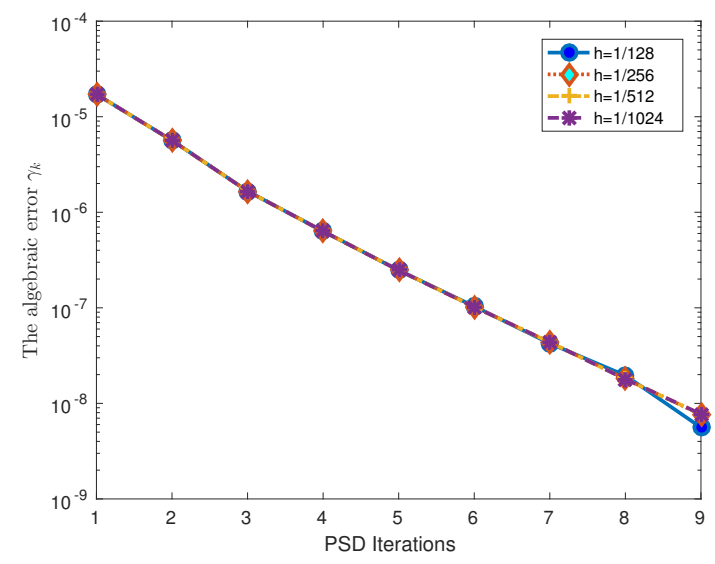

(a) $h$-independence: $p=4, s=0.01$ and $\varepsilon=0.03$.

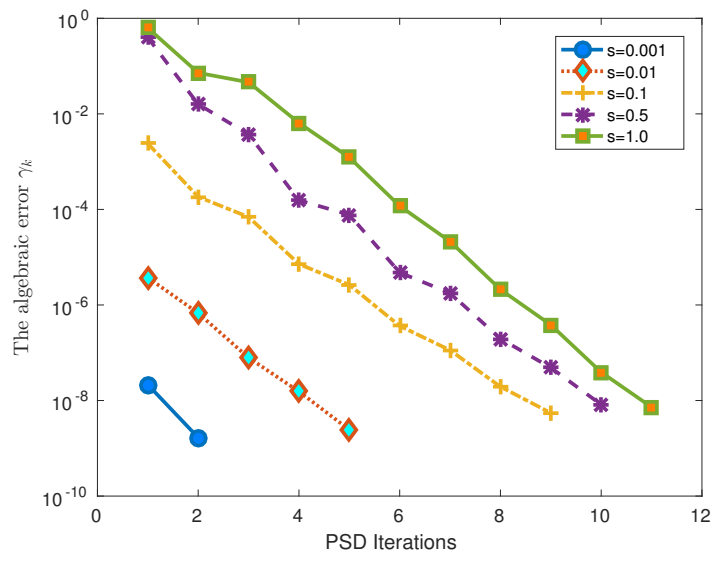

(c) s-dependence: $p=4, h=1 / 512$ and $\varepsilon=0.03$.

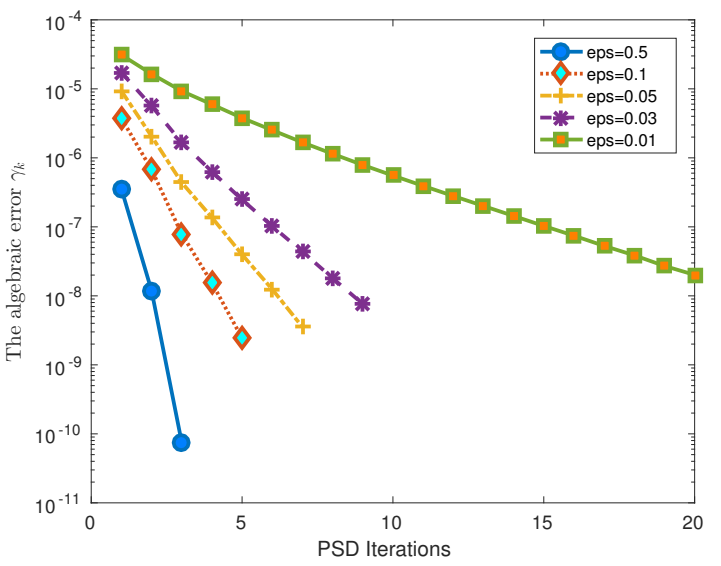

(b) $\varepsilon$-dependence: $p=4, s=0.01$ and $h=1 / 512$

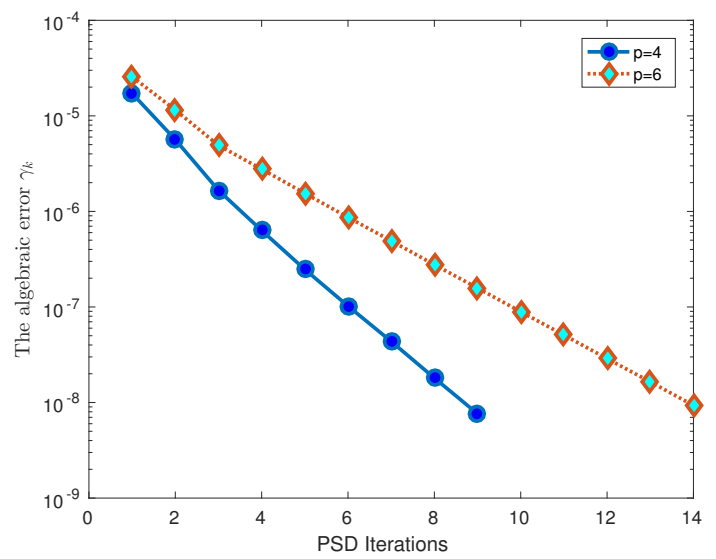

(d) $p$-dependence: $h=1 / 512, s=0.01$ and $\varepsilon=0.03$.

Figure 2: Complexity tests showing the solver performance for changing values of $h, \varepsilon, s$ and $p$. Parameters are given in the text.

\subsubsection{Long-time coarsening behavior for the thin film model with $p=4,6$}

Coarsening processes in thin film systems can take place on very long time scales [27]. In this subsection, we perform (now standard) long time behavior tests for $p=4,6$. Such test, which have been performed in many places, will confirm the expected coarsening rates and serve as benchmarks for our solver. See, for example, $[32,35]$. The initial data for the simulations are taken as essentially random:

$$
u_{i, j}^{0}=0.05 \cdot\left(2 r_{i, j}-1\right) \text {, }
$$

where the $r_{i, j}$ are uniformly distributed random numbers in $[0,1]$. Time snapshots of the evolution for the epitaxial thin film growth model with $p=4$ can be found in Figure 3. The coarsening rates for the $p=4$ case are given in Figure 4. These simulation results are consistent with earlier work on this topic in $[32,35,38]$, showing the surface roughness, $W$, grows like $t^{\frac{1}{3}}$ and the energy, $E$, decays like $t^{-\frac{1}{3}}$. We also present the numerical simulations for the epitaxial thin film growth model with $p=6$ in Figure 5. Notice in Figure 5 that the evolution process is significantly different from the process depicted in Figure 3. 


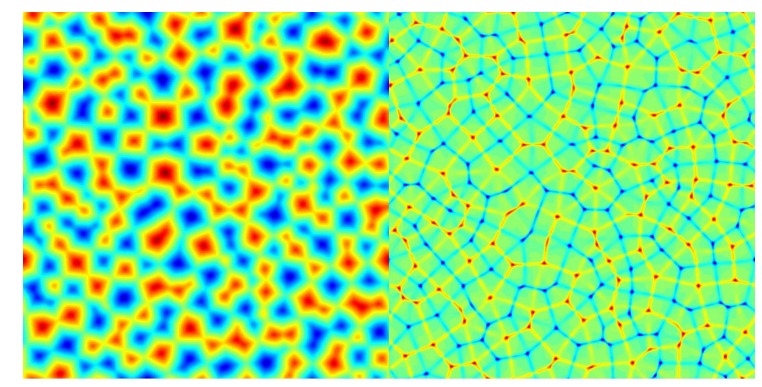

$t=10$

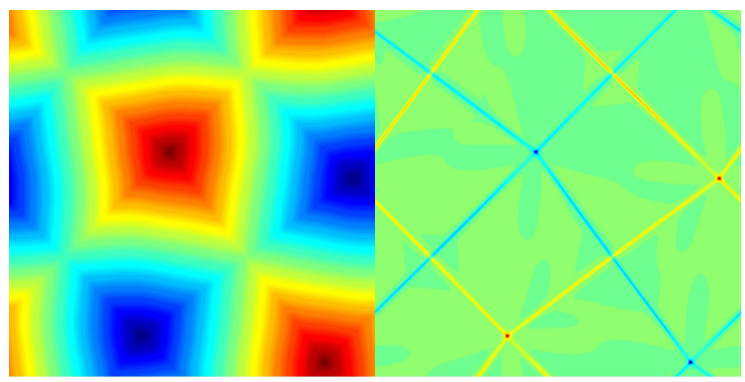

$t=3000$

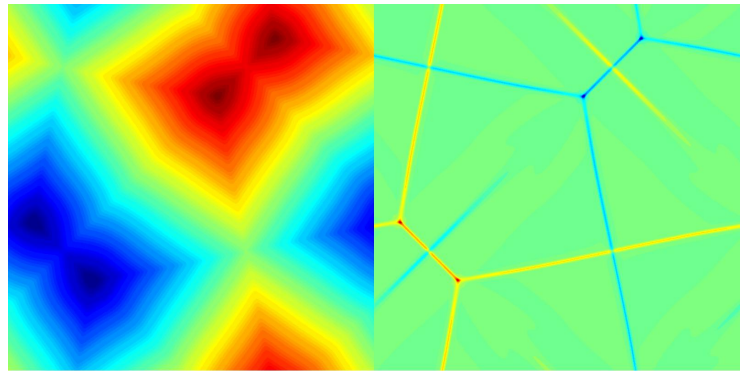

$t=8000$

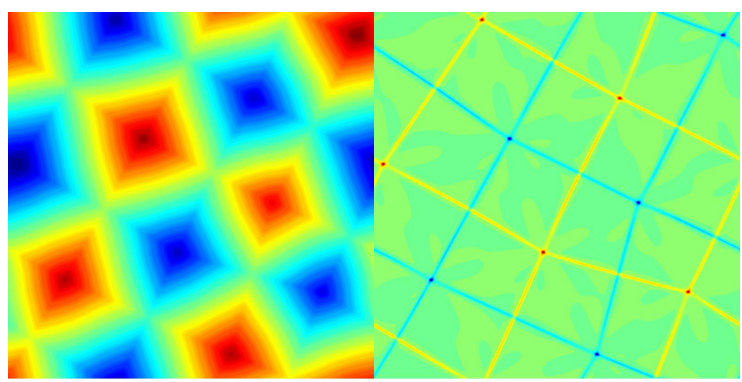

$t=1000$

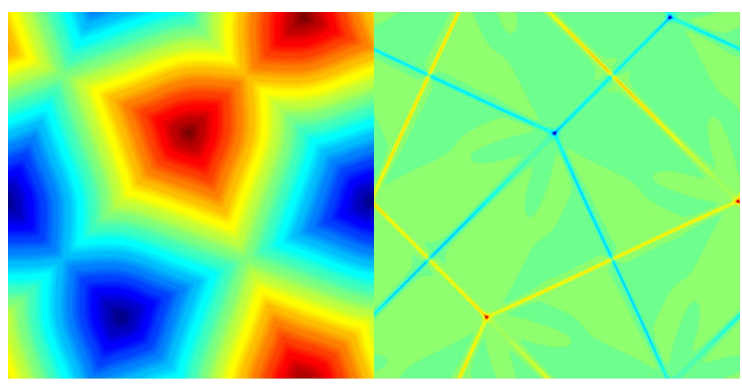

$t=6000$

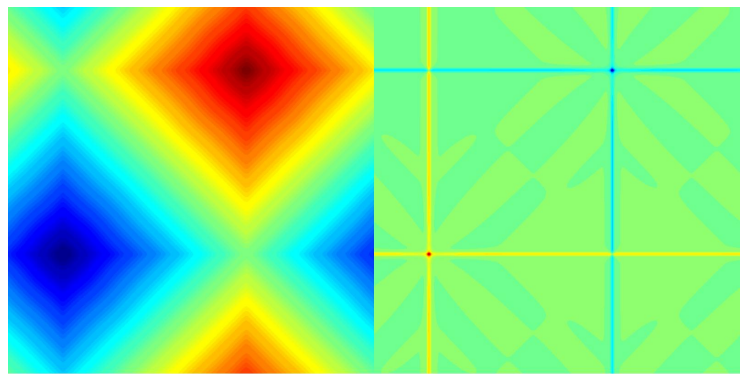

$t=10000$

Figure 3: Time snapshots of the evolution with PSD solver for the epitaxial thin film growth model with $p=4$ at $t=10,1000,3000,6000,8000$ and 10000 . Left: contour plot of $u$, Right: contour plot of $\Delta u$. The parameters are $\varepsilon=0.03, \Omega=[12.8]^{2}, s=0.01$. These simulation results are consistent with earlier work on this topic in $[32,35,38]$.

\subsection{Square Phase Field Crystal Model}

Suppose that $\Omega \subset \mathbb{R}^{d}, d=2,3$ is a rectangular domain. The energy of square phase field crystal (SPFC) model is given by $[15,19,20,30]$ :

$$
\mathcal{E}[u]=\int_{\Omega}\left\{\frac{\gamma_{0}}{2} u^{2}-\frac{\gamma_{1}}{2}|\nabla u|^{2}+\frac{\varepsilon^{2}}{2}|\Delta u|^{2}+\frac{1}{4}|\nabla u|^{4}\right\} d \mathbf{x},
$$

where $u: \Omega \rightarrow \mathbb{R}$ corresponds to the number density field of the atoms, and $\varepsilon>0, \gamma_{0}, \gamma_{1} \geq 0$ are parameters. The SPFC model is the $\mathrm{H}^{-1}$ gradient flow of this energy and is given by

$$
\partial_{t} u=\Delta w, \quad w:=\delta \mathcal{E}=\gamma_{0} u+\gamma_{1} \Delta u+\varepsilon^{2} \Delta^{2} u-\nabla \cdot\left(|\nabla u|^{2} \nabla u\right) .
$$

We propose the following fully-implicit, nonlinear convex decomposition scheme

$$
u^{n+1}-\Delta_{h} w^{n+1}=g, \quad s \gamma_{0} u^{n+1}-s \nabla_{h}^{v} \cdot\left(\left|\nabla_{h}^{v} u^{n+1}\right|^{2} \nabla_{h}^{v} u^{n+1}\right)+s \varepsilon^{2} \Delta_{h}^{2} u^{n+1}-w^{n+1}=f,
$$

where $g=u^{n}$ and $f=-\gamma_{1} \Delta_{h} u^{n}$. Using the techniques of $[35,37]$, we can prove that this scheme is unconditionally energy stable. The fully discrete scheme can also be rewritten in operator format as $\mathcal{N}_{h}\left[u^{n+1}\right]=f$, 


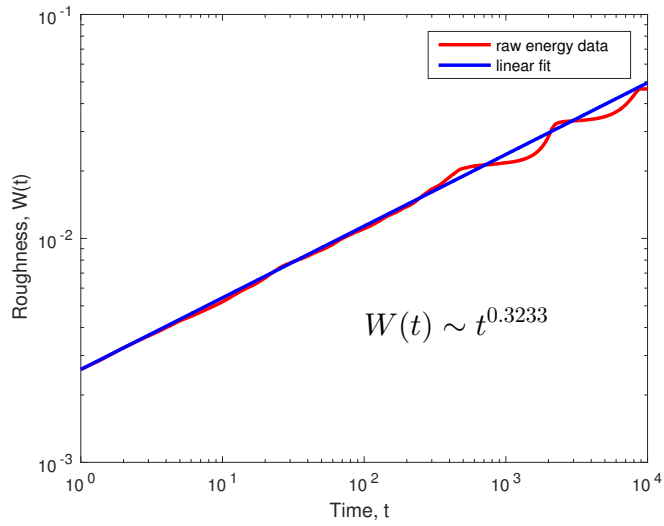

(a) Roughness evolution

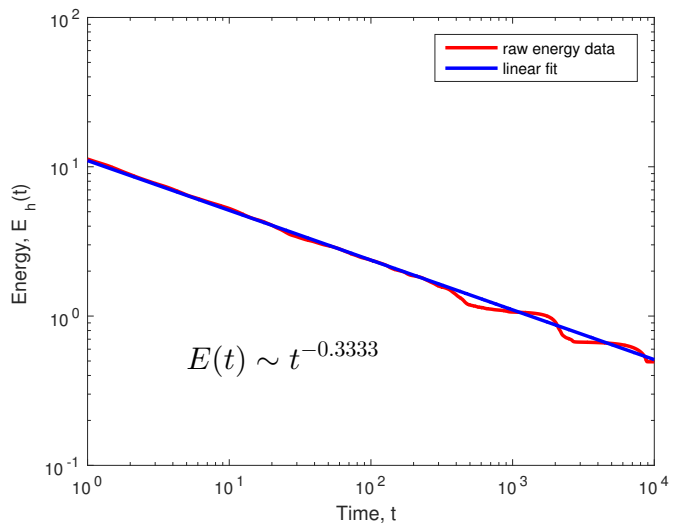

(b) Energy evolution

Figure 4: Log-log plot of Roughness and energy evolution for the simulation depicted in Figure 3.

where

$$
\mathcal{N}_{h}[\nu]:=s \gamma_{0} \nu+s \varepsilon^{2} \Delta_{h}^{2} \nu-s \nabla_{h}^{v} \cdot\left(\left|\nabla_{h}^{v} \nu\right|^{2} \nabla_{h}^{v} \nu\right)-T_{h}[-\nu+g] .
$$

We can shift the scheme from the affine space of solutions - whose elements $\nu$ satisfy $(\nu-\bar{g}, 1)_{2}=0$ - to the mean zero space, but this is not necessary for practical implementation. Otherwise, this scheme is in the scope of our theory, and, according to the prescription in Section 5.4, the pre-conditioner should be

$$
\mathcal{L}_{h}[\nu]:=s \gamma_{0} \nu-s \Delta_{h} \nu+s \varepsilon^{2} \Delta_{h}^{2} \nu-T_{h}[-\nu] .
$$

Given $u^{k} \in \mathcal{C}_{\text {per }}$, with $\left(u^{k}-\bar{g}, 1\right)_{2}=0$, we compute the search direction $d^{k} \in \mathcal{C}_{\text {per }}$ by solving the sixth order linear problem $\mathcal{L}_{h}\left[d^{k}\right]=f-\mathcal{N}_{h}\left[u^{k}\right]$ using FFT. Once $d^{k}$ is found, we perform the line-search: find $\alpha_{k} \in \mathbb{R}$ such that $q\left(\alpha_{k}\right)=0$, where

$$
q(\alpha)=\left(\mathcal{N}_{h}\left[u^{k}+\alpha d^{k}\right]-f, d^{k}\right)_{2} .
$$

After this, we update the approximation via $u^{k+1}=u^{k}+\alpha_{k} d^{k}$. As before, $q$ is a cubic polynomial (since $\left.p=4\right)$ whose coefficients can be precomputed. But this time, two of the coefficients involve the $T_{h}=-\Delta_{h}^{-1}$ operator. Specifically, for $q(\alpha)$ we need to compute

$$
\begin{aligned}
\left(\mathrm{T}_{h}\left[u^{k}-f+\alpha d^{k}\right], d^{k}\right)_{2} & =\left(\mathrm{T}_{h}\left[u^{k}-f\right], d^{k}\right)_{2}+\alpha\left(\mathrm{T}_{h}\left[d^{k}\right], d^{k}\right)_{2} \\
& =\left(u^{k}-f, \mathrm{~T}_{h}\left[d^{k}\right]\right)_{2}+\alpha\left(d^{k}, \mathrm{~T}_{h}\left[d^{k}\right]\right)_{2},
\end{aligned}
$$

where we have used the linearity and symmetry properties of the $T_{h}$ operator. These terms have only to be calculated once per line search, and can be efficiently computed using FFT. In fact, observe that we only need to compute $T_{h}\left[d^{k}\right]$, at the cost of a single FFT, per line search!

The 4-Laplacian term in (6.6) gives preference to rotationally invariant patterns with square symmetry. We perform a simple test showing the emergence of these patterns in this subsection. The initial data for those simulations are similar to (6.5), but we add nucleation sites at specific locations in the domain. The rest of the parameters are given by $\varepsilon=1.0 ; \lambda=\gamma_{0}=0.5 ; \gamma_{1}=2.0 ; \Omega=(0,100)^{2}$; and $s=0.01$. The time snapshots of the evolution by using the given parameters are presented in Figures 6 (one nucleation site) and 7 (four nucleation sites). These tests confirm the emergence of the rotationally invariant square-symmetry patterns in the density field $u$.

\section{Summary and Conclusions}

A preconditioned steepest descent (PSD) solver is proposed and analyzed for fourth and sixth-order regularized $\mathrm{p}$-Laplacian equations. Solution of the highly nonlinear finite difference equations are equivalent to the 


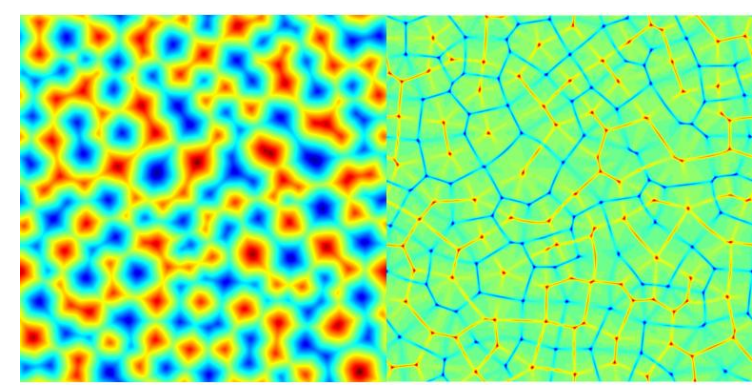

$t=10$

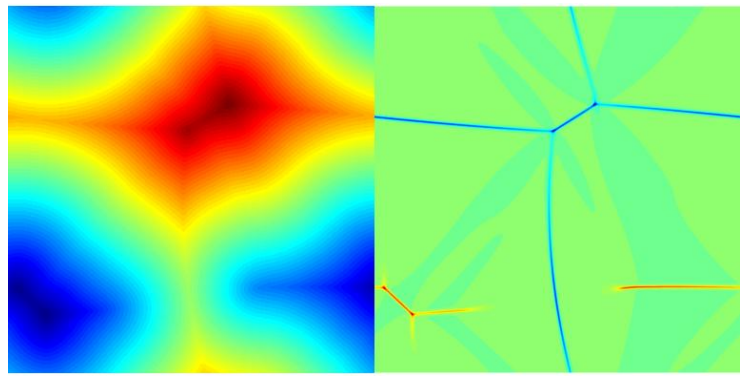

$t=3000$

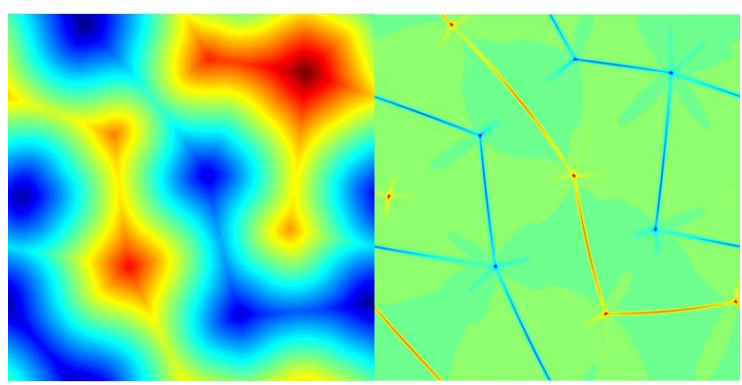

$t=1000$

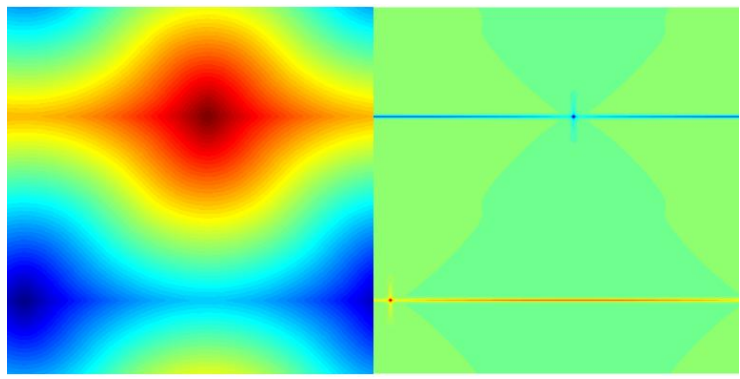

$t=6000$

Figure 5: Time snapshots of the evolution with PSD solver for the epitaxial thin film growth model with $p=6$ at $t=$ 10,1000,3000 and 6000. Left: contour plot of $u$, Right: contour plot of $\Delta u$. The parameters are $\epsilon=3.0 \times 10^{-2}, \Omega=$ $[12.8]^{2}, s=0.01$.

minimizations of associated strictly convex energies. The energy dissipation property of the PSD solver leads to a bound for the numerical solution at each iteration stage. This fact, coupled with an upper-bound for the second derivative of the energy with respect to the metric induced by the pre-conditioner, leads to a geometric convergence rate for our (PSD) solver, which is proved rigorously for both the continuous and discrete space cases. In the present setting the pre-conditioner is a linear, constant-coefficient, positive, and symmetric finite difference operator. The key to the efficiency of our method is that this pre-conditioner can be efficiently inverted using the FFT. Various numerical results are presented in this article, including a convergence test and a complexity analysis for the PSD solver, as well as long-time simulation results for the thin film epitaxy model with slope selection (both $p=4$ and $p=6$ ) and the square phase field crystal model.

Since we have shown rigorously that our equations result as the gradients of strictly convex functionals, it also possible to use Newton's method (or a quasi Newton's method) to solve the nonlinear equations. One will still obtain global convergence, and in fact, we expect the convergence rate to be faster than geometric. On the other hand, in the case of (5.1), say, Newton's method requires one to invert a complicated, nonconstant coefficient, fourth-order linear finite difference equation. One could not use FFT for the inversion of this operator but would have to design an efficient solver for this purpose. This is a non-trivial task. So, in summary, although the (quasi) Newton's method would give a faster convergence rate than the PSD solver - in particular, super-linear convergence $\left\|e^{k+1}\right\| \leq C\left\|e^{k}\right\|^{\beta}, \beta>1$, versus a geometric convergence rate - the PSD solver is, at least currently, much more efficient.

\section{Acknowledgments}

This work is supported in part by the grants NSF DMS-1418689 (C. Wang), NSF DMS-1418692 (S.M. Wise) and NSF DMS-1418784 (A.J. Salgado). 

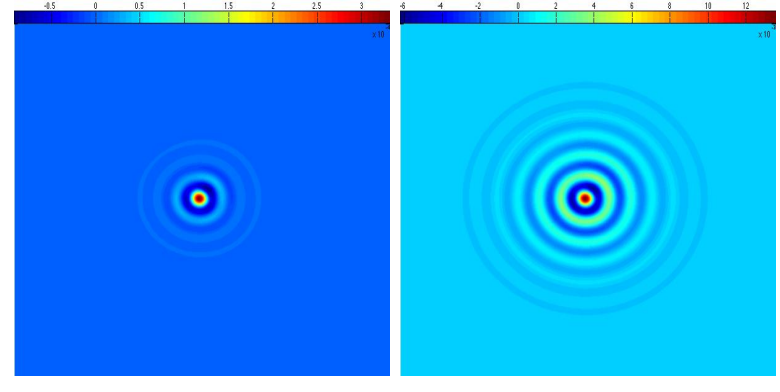

$t=1,10$

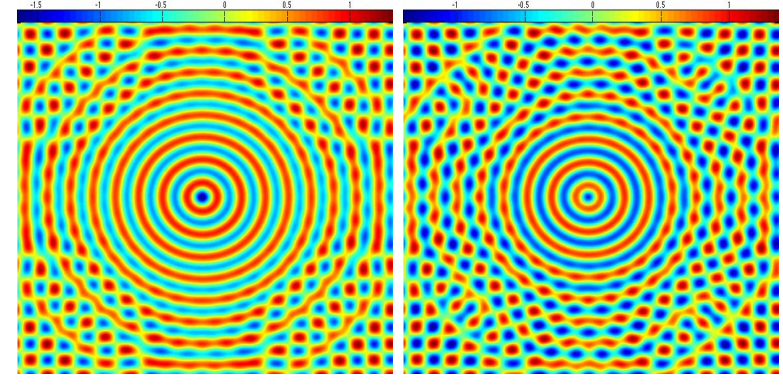

$t=60,80$

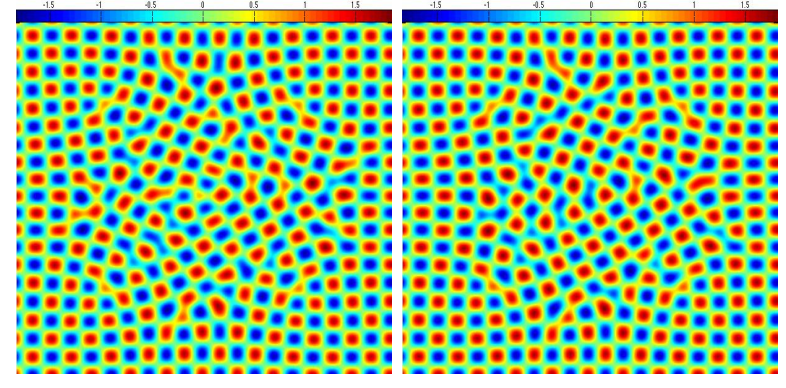

$t=500,1000$

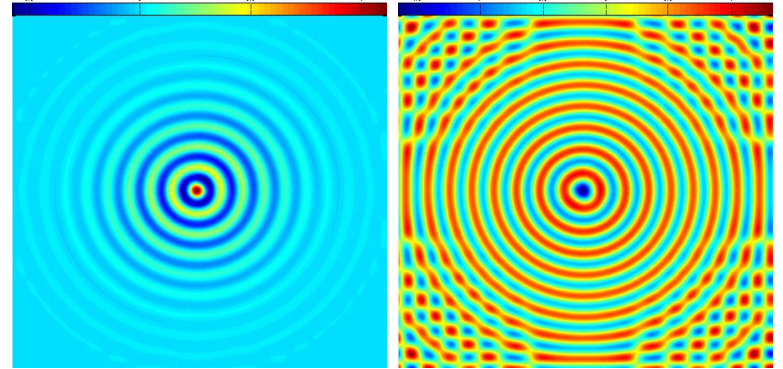

$t=20,40$

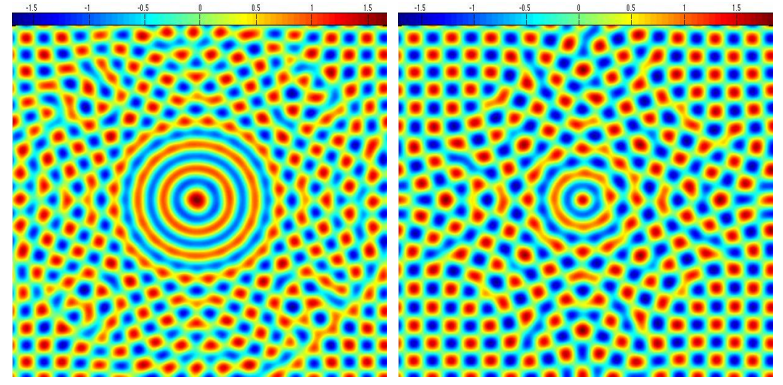

$t=100,200$

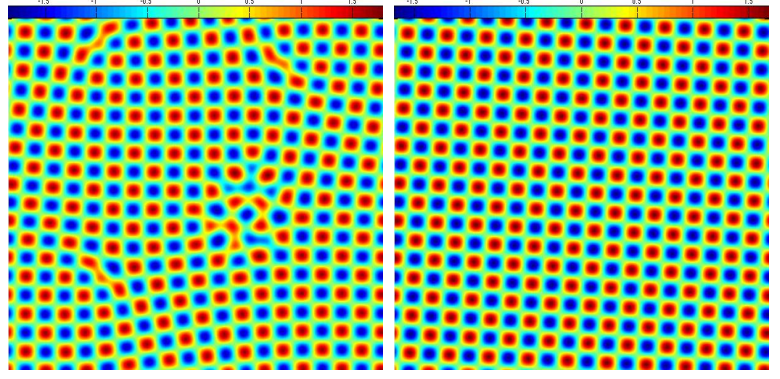

$t=5000,9000$

Figure 6: Time snapshots of the evolution with PSD solver for squared phase field crystal model at $t=$ $1,10,20,40,60,80,100,200,500,1000,5000$ and 9000 . The parameters are $\epsilon=1.0, \lambda=0.5, \gamma_{1}=2.0, \Omega=[100]^{2}$ and $s=0.01$.

\section{References}

[1] K. Atkinson and W. Han. Theoretical numerical analysis, volume 39. Springer, 2005.

[2] J.W. Barrett and W. Liu. Finite element approximation of the parabolic p-Laplacian. SIAM J. Numer. Anal., 31:413-428, 1994.

[3] R. Bermejo and J.A. Infante. A multigrid algorithm for the p-Laplacian. SIAM J. Sci. Comput., 21(5):17741789, 2000.

[4] J.P. Boyd. Chebyshev and Fourier spectral methods. Courier Corporation, 2001.

[5] J.W. Cahn and J.E. Hilliard. Free energy of a nonuniform system. I. Interfacial free energy. J. Chem. Phys., 28:258, 1958.

[6] K. Cheng, W. Feng, S. Gottlieb, and C. Wang. A Fourier pseudospectral method for the "Good" Boussinesq equation with second-order temporal accuracy. Numer. Methods Partial Differ. Equ., 31(1):202-224, 2015.

[7] K. Cheng, W. Feng, C. Wang, and S.M. Wise. A refined truncation error estimate for long stencil fourth order finite difference approximation and its application to the Cahn-Hilliard equation. in preparation, 2016. 


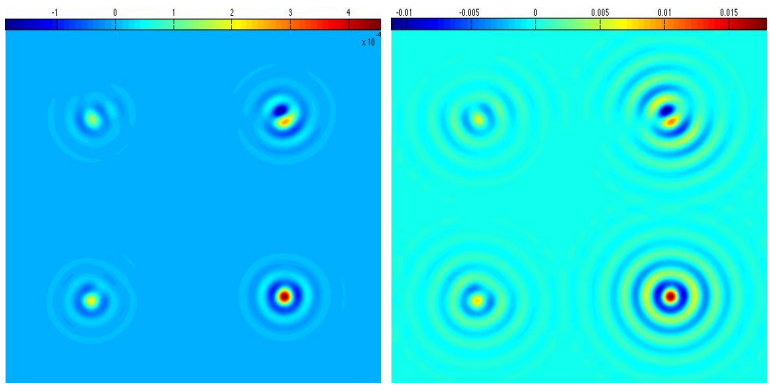

$t=1,10$

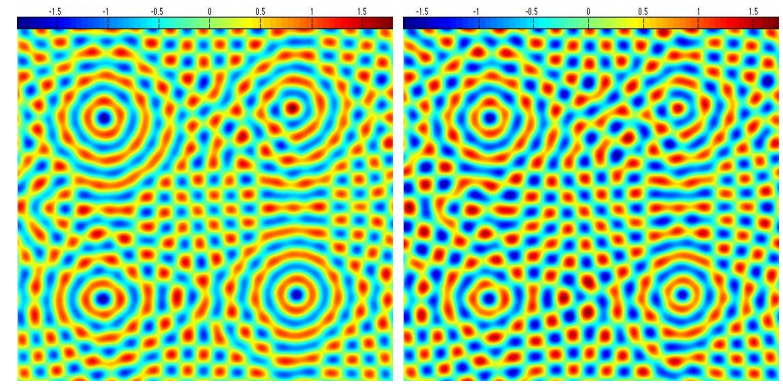

$$
t=60,80
$$

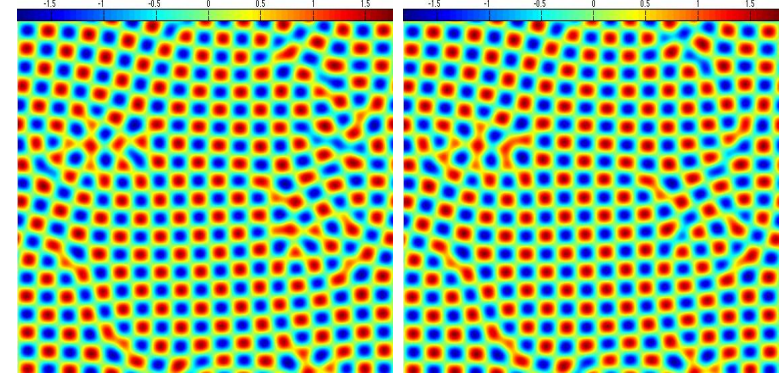

$t=600,800$

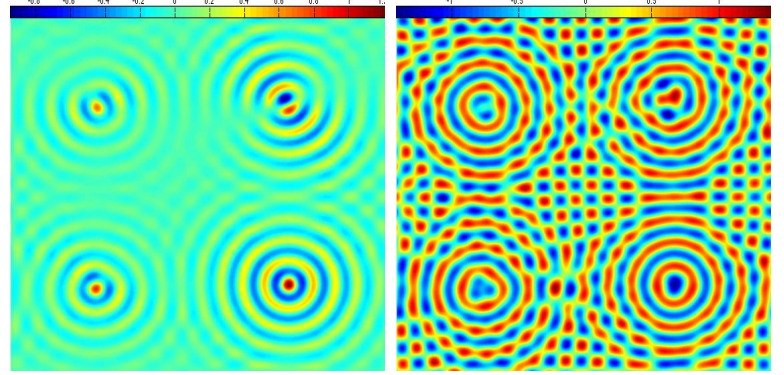

$t=20,40$

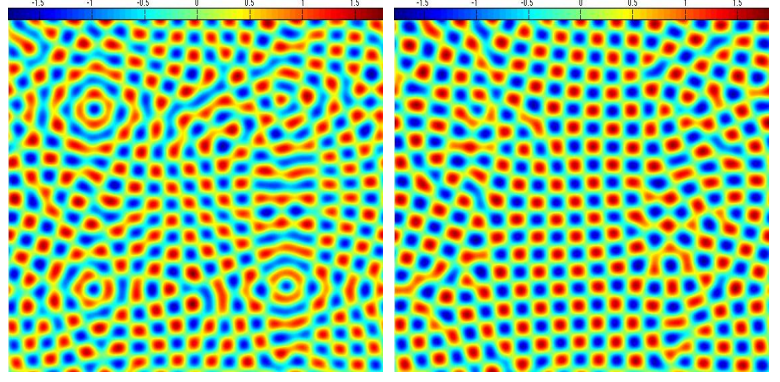

$t=100,200$

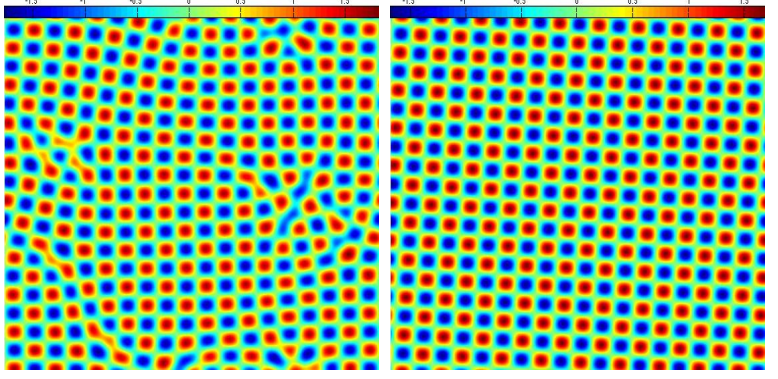

$t=1000,3000$

Figure 7: Time snapshots of the evolution with PSD solver for squared phase field crystal model at $t=$ $1,10,20,40,60,80,100,200,600,800,1000$ and 3000 . The parameters are $\epsilon=1.0, \lambda=0.5, \gamma_{1}=2.0, \Omega=[100]^{2}$ and $s=0.01$.

[8] A. Christlieb, J. Jones, K. Promislow, B. Wetton, and M. Willoughby. High accuracy solutions to energy gradient flows from material science models. J. Comput. Phys., 257, Part A:193 - 215, 2014.

[9] P.G. Ciarlet. Introduction to Numerical Linear Algebra and Optimisation. Cambridge University Press, New York, NY, USA, 1989.

[10] B. Cockburn and J. Shen. A hybridizable discontinuous Galerkin method for the p-Laplacian. SIAM J. Sci. Comput., 38(1):A545-A566, 2016.

[11] M.C. Cross and P.C. Hohenberg. Pattern formation outside of equilibrium. Rev. Mod. Phys., 65(3):851, 1993.

[12] A.E. Diegel, X. Feng, and S.M. Wise. Analysis of a mixed finite element method for a Cahn-HilliardDarcy-Stokes system. SIAM J. Numer. Anal., 53(1):127-152, 2015.

[13] A. Doelman, G. Hayrapetyan, K. Promislow, and B. Wetton. Meander and pearling of single-curvature bilayer interfaces in the functionalized Cahn-Hilliard equation. SIAM J. Math. Anal., 46(6):3640-3677, 2014. 
[14] I. Ekeland and R. Temam. Convex analysis and variational problems. SIAM, 1976.

[15] K.R. Elder, M. Katakowski, M. Haataja, and M. Grant. Modeling elastic and plastic deformations in nonequilibrium processing using phase field crystals. Phys. Rev. E, 70:051605, 2004.

[16] W. Feng, Z. Guan, J. S. Lowengrub, C. Wang, and S. M. Wise. An energy stable finite-difference scheme for functionalized Cahn-Hilliard equation and its convergence analysis. ArXiv e-prints 1610.02473, October 2016.

[17] W. Feng, A. J. Salgado, C. Wang, and S. M. Wise. Preconditioned steepest descent methods for some nonlinear elliptic equations involving p-Laplacian terms. ArXiv e-prints 1607.01475, July 2016.

[18] X. Feng and M. Neilan. Vanishing moment method and moment solution for second order fully nonlinear partial differential equations. J. Scient. Comp., 38(1):74-98, 2009.

[19] A.A Golovin and A.A. Nepomnyashchy. Disclinations in square and hexagonal patterns. Phys. Rev. E, 67:056202, 2003.

[20] J.A.M. Hernández, F.G. Castañeda, and J.A.M. Cadenas. Formation of square patterns using a model alike Swift-Hohenberg. In 2014 11th International Conference on Electrical Engineering, Computing Science and Automatic Control (CCE), pages 1-6. IEEE, 2014.

[21] J.S. Hesthaven, S. Gottlieband, and D. Gottlieb. Spectral methods for time-dependent problems, volume 21. Cambridge University Press, 2007.

[22] R.B. Hoyle. Steady squares and hexagons on a subcritical ramp. Phys. Rev. E, 51(1):310, 1995.

[23] R.B. Hoyle. Pattern formation: an introduction to methods. Cambridge University Press, 2006.

[24] Z. Hu, S.M. Wise, C. Wang, and J.S. Lowengrub. Stable and efficient finite-difference nonlinear-multigrid schemes for the phase-field crystal equation. J. Comput. Phys., 228:5323-5339, 2009.

[25] Y. Huang, R. Li, and W. Liu. Preconditioned descent algorithms for p-Laplacian. J. Sci. Comput., 32(2):343-371, 2007.

[26] A.V. Knyazev and I. Lashuk. Steepest descent and conjugate gradient methods with variable preconditioning. SIAM J. Matrix Anal. Appl., 29(4):1267-1280, 2007.

[27] R.V. Kohn. Energy-driven pattern formation. In International Congress of Mathematicians, volume 1, pages 359-383, 2006.

[28] B. Li and J. Liu. Epitaxial growth without slope selection: energetics, coarsening, and dynamic scaling. J. Nonlinear Sci., 14(5):429-451, 2004.

[29] W. Liu and N. Yan. Quasi-norm local error estimators for p-Laplacian. SIAM J. Numer. Anal., 39(1):100127, 2001.

[30] D.J. Lloyd, B.Sandstede, D. Avitabile, and A.R. Champneys. Localized hexagon patterns of the planar Swift-Hohenberg equation. SIAM J. Appl. Dyn. Syst., 7(3):1049-1100, 2008.

[31] R.L. Pego. Front migration in the nonlinear Cahn-Hilliard equation. Proc. R. Soc. Lond. A, 422:261-278, 1989.

[32] J. Shen, C. Wang, X. Wang, and S.M. Wise. Second-order convex splitting schemes for gradient flows with Ehrlich-Schwoebel type energy: application to thin film epitaxy. SIAM J. Numer. Anal., 50(1):105-125, 2012.

[33] J. Shen and X. Yang. Numerical approximations of Allen-Cahn and Cahn-Hilliard equations. Discrete Contin. Dyn. Syst., 28:1669-1691, 2010. 
[34] X. Tai and J. Xu. Global and uniform convergence of subspace correction methods for some convex optimization problems. Math. Comp., 71(237):105-124, 2002.

[35] C. Wang, X. Wang, and S.M. Wise. Unconditionally stable schemes for equations of thin film epitaxy. Discrete Contin. Dyn. Syst., 28(1):405-423, 2010.

[36] C. Wang and S.M. Wise. An energy stable and convergent finite-difference scheme for the modified phase field crystal equation. SIAM J. Numer. Anal., 49(3):945-969, 2011.

[37] S.M. Wise, C. Wang, and J. Lowengrub. An energy stable and convergent finite-difference scheme for the phase field crystal equation. SIAM J. Numer. Anal., 47:2269-2288, 2009.

[38] C. Xu and T. Tang. Stability analysis of large time-stepping methods for epitaxial growth models. SIAM J. Numer. Anal., 44(4):1759-1779, 2006.

[39] G. Zhou and C. Feng. The steepest descent algorithm without line search for p-Laplacian. Appl. Math. Comput., 224:36-45, 2013.

[40] G. Zhou, Y. Huang, and C. Feng. Preconditioned hybrid conjugate gradient algorithm for p-Laplacian. Int. J. Numer. Anal. Model., 2:123-130, 2005. 